\title{
From HAG to DAG: derived moduli stacks
}

\author{
Bertrand Toën \\ Laboratoire J. A. Dieudonné \\ UMR CNRS 6621 \\ Université de Nice Sophia-Antipolis \\ France
}

\author{
Gabriele Vezzosi \\ Dipartimento di Matematica \\ Università di Bologna \\ Italy
}

October 2002

\begin{abstract}
These are expanded notes of some talks given during the fall 2002, about homotopical algebraic geometry with special emphasis on its applications to derived algebraic geometry and derived deformation theory.

We use the general framework developed in [HAG-I], and in particular the notions of model topology, model sites and stacks over them, in order to define various derived moduli functors and study their geometric properties. We start by defining the model category of $D$-stacks, with respect to an extension of the étale topology to the category of commutative differential graded algebras, and we show that its homotopy category contains interesting objects, such as schemes, algebraic stacks, higher algebraic stacks, dg-schemes, etc. We define the notion of geometric $D$ stacks and present some related geometric constructions $(\mathcal{O}$-modules, perfect complexes, $K$-theory, derived tangent stacks, cotangent complexes, various notions of smoothness, etc.). Finally, we define and study the derived moduli problems classifying local systems on a topological space, vector bundles on a smooth projective variety, and $A_{\infty}$-categorical structures. We state geometricity and smoothness results for these examples. The proofs of the results presented in this paper will be mainly given in [HAG-II].
\end{abstract}

Key words: Moduli spaces, stacks, dg-schemes, deformation theory, $A_{\infty}$-categories.

\section{Contents}

1 Introduction $\quad 2$

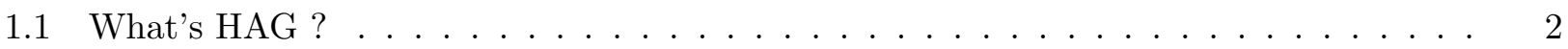

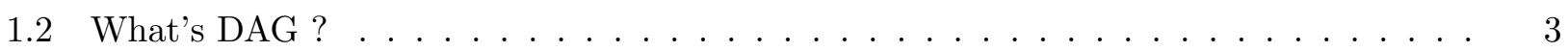

1.3 General comments on deriving moduli functors $\ldots \ldots \ldots \ldots$

$\begin{array}{lll}2 & \text { The model category of } D \text {-stacks } & 7\end{array}$

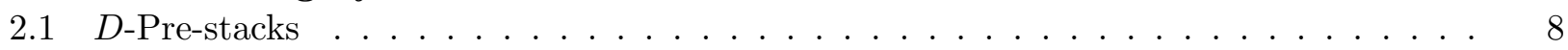

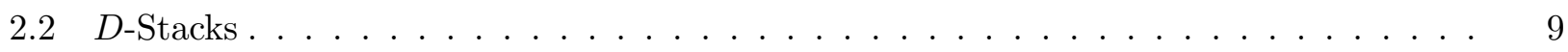

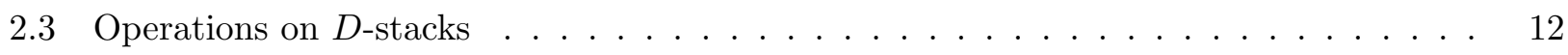




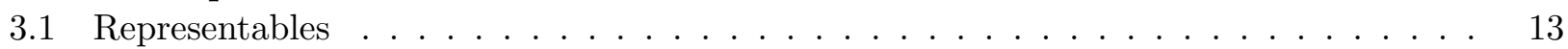

3.2 Stacks vs. $D$-stacks $\ldots \ldots \ldots \ldots \ldots \ldots \ldots \ldots \ldots \ldots \ldots$

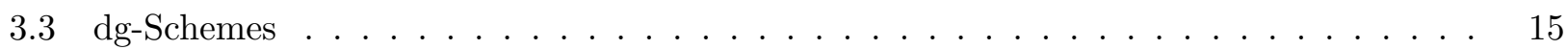

3.4 The $D$-stack of $G$-torsors $\ldots \ldots \ldots \ldots \ldots \ldots \ldots \ldots$

4 The geometry of $D$-stacks $\quad 17$

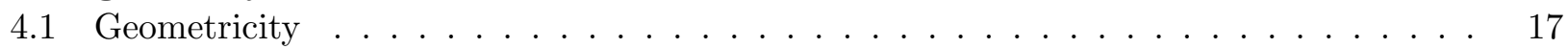

4.2 Modules, linear $D$-stacks and $K$-theory $\ldots \ldots \ldots \ldots \ldots \ldots$

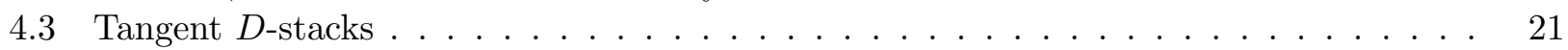

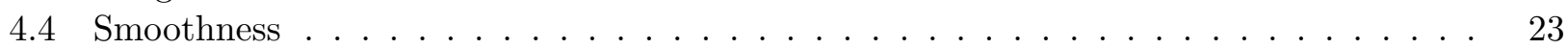

5 Further examples $\quad \mathbf{2 4}$

5.1 Local systems on a topological space . . . . . . . . . . . . . . . . . 25

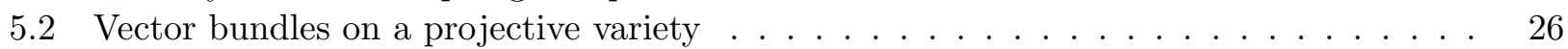

5.3 Algebras and $A_{\infty}$-categorical structures $\ldots \ldots \ldots \ldots \ldots \ldots$

\section{Introduction}

These are expanded notes of some talks given during Fall 2002 about homotopical algebraic geometry with special emphasis on its applications to derived algebraic geometry and derived deformation theory. We have omitted proofs that will appear mainly in [HAG-II]. The main purpose of this work is to present in a somehow informal way the category of $D$-stacks and to give some examples of derived moduli spaces as $D$-stacks.

We would like to thank the organizers of the conferences "Axiomatic and enriched homotopy theory" (Cambridge, September 2002) and "Intersection theory and moduli" (Trieste, September 2002), in which some of the material in the present note has been reported. We would also like to thank S. Müller-Stach, H. Esnault and E. Viehweg for inviting us to lecture on our work at a DFG-Schwerpunkt "Globale Methoden in der Komplexen Geometrie" in Essen and the Research in Pairs program at the Matematisches Forschunginstitut Oberwolfach for providing the excellent working conditions in which this paper was written.

\subsection{What's HAG ?}

Homotopical Algebraic Geometry (or HAlgebraic Geometry, or simply HAG) was conceived as a framework to talk about schemes in a context where affine objects are in one-to-one correspondence with commutative monoid-like objects in a base symmetric monoidal model category.

This general definition might seem somewhat obscure, so we'd rather mention the most important examples of base symmetric monoidal model category, and the corresponding notions of commutative monoid-like objects. In each of the following situations, HAG will provide a context in which one can do algebraic geometry (and in particular, talk about schemes, algebraic spaces, stacks ...), hence giving rise to various geometries.

1. The model category $A b$ of abelian groups (with its trivial model structure) and the tensor product of abelian groups. Commutative monoid objects are commutative rings. The corresponding geometry is the usual, Grothendieck-style algebraic geometry. 
2. The model category $\operatorname{Mod}(\mathcal{O})$ of $\mathcal{O}$-modules over some ringed site (with the trivial model structure) and the tensor product of $\mathcal{O}$-modules. Commutative monoid objects are sheaves of commutative $\mathcal{O}$-algebras. The corresponding geometry is called relative algebraic geometry, and was introduced and studied in [Ha, De].

3. The model category $C(k)$ of complexes over some ring $\mathrm{k}$ and the tensor product of complexes (see $[\mathrm{Ho}, \S 2.3])$. Commutative monoid-like objects are $E_{\infty}$-algebras over $k$ ([Kr-Ma]). The corresponding geometry is the so-called derived algebraic geometry that we are going to discuss in details in this paper, and for which one possible avatar is the theory of dg-schemes and dg-stacks of [Ci-Ka1, Ci-Ka2].

4. The model category $S p$ of symmetric spectra and the smash product (see [Ho-Sh-Sm]), or equivalently the category of $\mathbb{S}$-modules (see $[\mathrm{EKMM}]$ ). Commutative monoid-like objects are $E_{\infty}$-ring spectra, or commutative $\mathbb{S}$-algebras. We call the corresponding geometry brave new algebraic geometry, quoting the expression brave new algebra introduced by F. Waldhausen (for more details on the subject, see e.g. [Vo]).

5. The model category Cat of categories and the direct product (see, e.g. [Jo-Ti]). Commutative monoid-like objects are symmetric monoidal categories. The corresponding geometry does not have yet a precise name, but could be called 2-algebraic geometry, since vector bundles in this setting will include both the notion of 2-vector spaces (see [Ka-Vo]) and its generalization to 2-vector bundles.

For the general framework, we refer the reader to [HAG-I, HAG-II]. The purpose of the present note is to present one possible incarnation of HAG through a concrete application to derived algebraic geometry (or "DAG" for short).

\subsection{What's DAG ?}

Of course, the answer we give below is just our own limited understanding of the subject.

As far as we know, the foundational ideas of derived algebraic geometry (whose infinitesimal theory is also referred to as derived deformation theory, or "DDT" for short) were introduced by P. Deligne, V. Drinfel'd and M. Kontsevich, for the purpose of studying the so-called derived moduli spaces. One of the main observations was that certain moduli spaces were very singular and not of the expected dimension, and according to the general philosophy this was considered as somehow unnatural (see the hidden smoothness philosophy presented in [Kol]). It was therefore expected that these moduli spaces are only truncations of some richer geometric objects, called the derived moduli spaces, containing important additional structures making them smooth and of the expected dimension. In order to illustrate these general ideas, we present here the fundamental example of the moduli stack of vector bundles (see the introductions of [Ci-Ka1, Ci-Ka2, Ka1] for more motivating examples as well as philosophical remarks).

Let $C$ be a smooth projective curve (say over $\mathbb{C}$ ), and let us consider the moduli stack $\underline{V e c t}{ }_{n}(C)$ of rank $n$ vector bundles on $C$ (here $\operatorname{Vect}_{n}(C)$ classifies all vector bundles on $C$, not only the semistable or stable ones). The stack $\operatorname{Vect}_{n}(C)$ is known to be an algebraic stack (in the sense of Artin). Furthermore, if $E \in \underline{V e c t}_{n}(C)(\mathbb{C})$ is a vector bundle on $C$, one can easily compute the stacky tangent space of $\underline{V e c t}_{n}(C)$ at the point $E$. This stacky tangent space is actually a complex of $\mathbb{C}$-vector spaces concentrated in degrees $[-1,0]$, which is easily seen to be quasi-isomorphic to the complex $C^{*}\left(C_{Z a r}, \underline{E n d}(E)\right)[1]$ of Zariski cohomology of $C$ with coefficient in the vector bundle $\underline{E n d}(E)=$ 
$E \otimes E^{*}$. Symbolically, one writes

$$
T_{E} \underline{\operatorname{Vect}}(C) \simeq H^{1}(C, \underline{\operatorname{End}}(E))-H^{0}(C, \underline{\operatorname{End}}(E)) .
$$

This implies in particular that the dimension of $T_{E} \underline{V e c t}(C)$ is independent of the point $E$, and is equal to $n^{2}(g-1)$, where $g$ is the genus of $C$. The conclusion is then that the stack $\operatorname{Vect}_{n}(C)$ is smooth of dimension $n^{2}(g-1)$.

Let now $S$ be a smooth projective surface, and $\underline{V e c t}_{n}(S)$ the moduli stack of vector bundles on $S$. Once again, $\underline{\operatorname{Vect}}_{n}(S)$ is an algebraic stack, and the stacky tangent space at a point $E \in \underline{V e c t}_{n}(S)(\mathbb{C})$ is easily seen to be given by the same formula

$$
T_{E} \underline{\operatorname{Vect}}_{n}(S) \simeq H^{1}(S, \underline{\operatorname{End}}(E))-H^{0}(S, \underline{\operatorname{End}}(E)) .
$$

Now, as $H^{2}(S, \underline{E n d}(E))$ might jump when specializing $E$, the dimension of $T_{E} \operatorname{Vect}(S)$, which is $h^{1}(S, \underline{E n d}(E))-h^{0}(S, \underline{E n d}(E))$, is not locally constant and therefore the stack $\underline{\operatorname{Vect}}_{n}(S)$ is not smooth anymore.

As recalled above, the main idea of derived algebraic geometry is that usual moduli spaces are in fact truncations of suitable "graded" moduli spaces, called derived moduli spaces; for example, $\underline{V e c t}_{n}(S)$ should be only the truncation of a richer object $\mathbb{R} \underline{V e c t}_{n}(S)$, called the derived moduli stack of vector bundles on $S$. This derived moduli stack, whatever it may be, should be such that its tangent space at a point $E$ is the whole complex $C^{*}(S, \underline{\operatorname{End}}(E))[1]$, or in other words,

$$
T_{E} \mathbb{R} \underline{\operatorname{Vect}}_{n}(S) \simeq-H^{2}(S, \underline{\operatorname{End}}(E))+H^{1}(S, \underline{\operatorname{End}}(E))-H^{0}(S, \underline{\operatorname{End}}(E)) .
$$

The dimension of its tangent space at $E$ is then expected to be $-\chi(S, \underline{E n d}(E))$, and therefore locally constant. Hence, the object $\mathbb{R} \underline{V e c t}_{n}(S)$ is expected to be smooth.

Remark 1.1 Another, very similar but probably more striking example is given by the moduli stack of stable maps, introduced in [Ko1]. A consequence of the expected existence of the derived moduli stack of stable maps is the presence of a virtual structure sheaf giving rise to a virtual fundamental class (see $[\mathrm{Be}-\mathrm{Fa}]$ ). The importance of such constructions in the context of Gromov-Witten theory shows that the extra information contained in derived moduli spaces is very interesting and definitely geometrically meaningful.

In the above example of the stack of vector bundles, the tangent space of $\mathbb{R} V e c t_{n}(S)$ is expected to be a complex concentrated in degree $[-1,1]$. More generally, tangent spaces of derived moduli (1-)stacks should be complexes concentrated in degree [-1, $\infty$ [ (see [Ci-Ka1]). It is therefore pretty clear that in order to make sense of an object such as $\mathbb{R} \underline{V e c t}{ }_{n}(S)$, schemes and algebraic stacks are not enough, and one should look for a more general definition of spaces. Now, since a smooth variety $X$, locally at a point $x \in X$ looks like $\operatorname{Spec} \operatorname{Sym}\left(T_{X, x}^{*}\right)$, where $T_{X, x}^{*}$ is the dual to the tangent space of $X$ at $x$, one may conclude that locally derived moduli spaces should look like $\operatorname{Spec}(\operatorname{Sym}(C))$, where now $C$ is a (bounded below) complex of vector spaces (and $S p e c$ has to be properly defined). Therefore the affine models of derived algebraic geometry should be commutative differential (negatively) graded algebras, cdga's for short. This leads to the following general question.

Problem: Provide a framework in which derived moduli stacks can actually be constructed. In particular, construct the derived moduli stack of vector bundles $\mathbb{R}$ Vect $(S)$ discussed above.

Several construction of formal derived moduli spaces have appeared in the literature (see for example [Ko-So, So]), a general framework for formal DAG have been developed by V. Hinich in 
[Hin2], and pro-representability questions were investigated by Manetti in [Man]. So, in a sense, the formal theory has already been worked out, and what remains of the problem above is an approach to global DAG.

A first approach to the global theory was proposed by M. Kapranov and I. Ciocan-Fontanine, and is based on the theory of $d g$-schemes or more generally of $d g$-stacks (see [Ci-Ka1, Ci-Ka2]). A dg-scheme is, roughly speaking, a scheme together with an enrichment of its structural sheaf into commutative differential graded algebras. This enriched structural sheaf is precisely the datum encoding the derived information. This approach has been very successful, and many interesting derived moduli spaces (or stacks) have already been constructed as dg-schemes (e.g. the derived version of the Hilbert scheme, of the Quot scheme, of the stack of stable maps, and of the stack of local systems on a space have been defined in [Ka2, Ci-Ka1, Ci-Ka2]). However, this approach have encountered two major problems, already identified in [Ci-Ka2, 0.3].

1. The definition of dg-schemes and dg-stacks seems too rigid for certain purposes. By definition, a dg-scheme is a space obtained by gluing commutative differential graded algebras for the Zariski topology. It seems however that certain constructions really require a weaker notion of gluing, as for example gluing differential graded algebras up to quasi-isomorphisms (and a weaker topology).

2. The notion of dg-schemes is not very well suited with respect to the functorial point of view, as representable functors would have to be defined on the derived category of dg-schemes (i.e. the category obtained by formally inverting quasi-isomorphisms of dg-schemes), which seems difficult to describe and to work with. As a consequence, the derived moduli spaces constructed in [Ka2, Ci-Ka1, Ci-Ka2] do not arise as solution to natural derived moduli problems, and are constructed in a rather ad-hoc way.

The first of these difficulties seems of a technical nature, whereas the second one seems more fundamental. It seems a direct consequence of these two problems that the derived stack of vector bundles still remains to be constructed in this framework (see [Ka1] and [Ci-Ka1, Rem. 4.3.8]).

It is the purpose of this note to show how HAG might be applied to provide a framework for DAG in which problems (1) and (2) hopefully disappear. We will show in particular how to make sense of various derived moduli functors whose representability can be proved in many cases.

\subsection{General comments on deriving moduli functors}

To conclude this introduction we would like to discuss, from a general perspective, the problem of derivation of moduli functors, with the aim of at least making explicit some general features shared by the examples that will be presented in the main body of the paper (especially in Section 5). We will limit ourselves to the kind moduli functors that arise in algebraic geometry, leaving to the homotopy theorist the task of adapting our general discussion below to moduli problems arising in algebraic topology (such as the moduli space of $E_{\infty}$-ring spectrum structures on a given spectrum).

Suppose $M:(A f f)^{\mathrm{op}}=(\mathbb{C}-a l g) \longrightarrow$ Set is a functor arising from some algebro-geometric moduli problem e.g., the problem of classifying isomorphism classes of families of (pointed) curves of a given genus. Very often, the moduli functor $M$ is not representable and only admits a coarse moduli space. The theory of stacks in groupoids was originally invented to correct this annoyance, by looking at natural extensions of $M$, i.e. to functors $\mathcal{M}_{1}$, from algebras to groupoids, such that the following 
diagram commutes

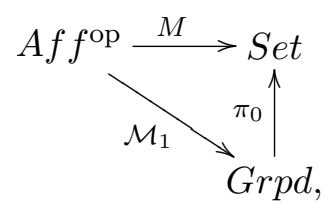

Here the vertical arrow assigns to a given groupoid its set of isomorphisms classes of objects. Of course, the point of the theory of stacks in groupoids is precisely to develop a geometry on this kind of functors. More generally, other natural higher moduli problems are not representable even when considered as stacks in groupoids (e.g. the 2-stack of perfect complexes of length 1 or the 2-stack of linear categories); the theory of higher stacks precisely says that one should consider $M$ extended as follows

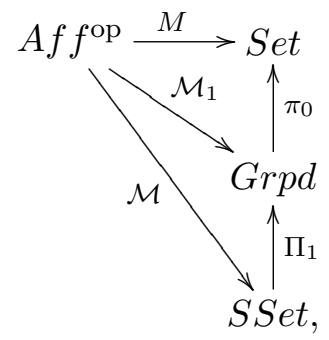

where $\Pi_{1}$ is the fundamental groupoid functor. The notion of geometric $n$-stacks of [S1] can then be used in order to set up a reasonable geometry over these kind of objects, in pretty much the same way one does geometry over algebraic stacks in groupoids.

The idea of derived algebraic geometry, as already explained above, is to seek for derived extensions of $M, \mathcal{M}_{1}$ and $\mathcal{M}$ i.e. to extend not (only) the target category of this functors but more crucially the source category in a "derived" direction. More precisely, if we denote by $D-A f f$ the opposite category of cdga's over $\mathbb{C}$, we define a derived extension as a functor $\mathbb{R} \mathcal{M}:(D-A f f)^{\text {op }} \longrightarrow S S e t s$ making the following diagram commute

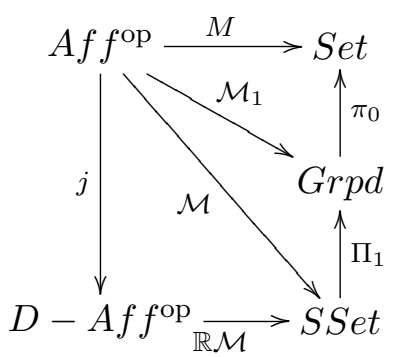

where $j$ denotes the natural inclusion (a $\mathbb{C}$-algebra viewed as a cdga concentrated in degree zero). The above diagram shows that, for any derived extension $\mathbb{R} \mathcal{M}$, we have

$$
\pi_{0} \mathbb{R} \mathcal{M}(j(\operatorname{Spec} R)) \simeq M(\operatorname{Spec} R)
$$

and moreover

$$
\Pi_{1} \mathbb{R} \mathcal{M}(j(\operatorname{Spec} R)) \simeq \mathcal{M}_{1}(\operatorname{Spec} R)
$$

for any commutative $\mathbb{C}$-algebra $R$. In other words, the 0 -truncation of $\mathbb{R} \mathcal{M}$ gives back $M$ when restricted to the image of $j$, while its 1 -truncation gives back $\mathcal{M}_{1}$.

What about the existence or uniqueness of a derived extension $\mathbb{R} \mathcal{M}$ ? First of all, extensions always exists: one can take the trivial one given by the functor $i$ of $\S 3.2$; however this extension is not only non-unique but also does not give, in most cases, the expected answer. However, there is no canonical choice for an extension which could be nicer than others and the choice of the extended moduli 
functor $\mathbb{R} \mathcal{M}$ highly depends on the geometrical meaning of the original moduli functor $\mathcal{M}$. Here is an example. Let $S^{2}$ be the 2-dimensional sphere, and let us consider $\mathcal{M}_{1}:=\underline{L o c}_{n}\left(S^{2}\right)$, the moduli stack of rank $n$ local systems on $S^{2}$. We clearly have $\mathcal{M}_{1} \simeq B G l_{n}$ and if one uses this isomorphism as an identification, then a reasonable extension of $\mathcal{M}_{1}$ is simply $i B G l_{n} \simeq \mathbb{R} B G l_{n}$ as it will be described in $\S 3.4$. However, if one remembers that $\mathcal{M}_{1}$ is $\underline{L o c}_{n}\left(S^{2}\right)$, then the correct (or at least expected) extension is $\mathbb{R} \underline{\operatorname{Loc}}_{n}\left(S^{2}\right)$ presented below in Theorem 5.1. These two extensions are very different. This shows that the expected extension $\mathbb{R} \mathcal{M}$ depends very much on the way we think of the original moduli problem $\mathcal{M}$. Another example of the existence of multiple choices can be found in [Ci-Ka2], in which the derived Hilbert dg-scheme is not the same as the derived Quot $_{\mathcal{O}}$ dg-scheme.

Nevertheless, the derived extension of a moduli functor that typically occurs in algebraic geometry, is expected to satisfy certain properties and this gives some serious hints to the correct answer. First of all, due to the fact that the derived deformation theory (and the corresponding formal theory) of the moduli problem has already been studied (see [Hin2, Ko-So, So], to quote a few), one knows a priori what is the expected derived tangent stack $T \mathbb{R} \mathcal{M}$ (or, at least, the disembodied derived tangent complexes at the points, the $\left(\Omega_{\mathbb{R} \mathcal{M}, x}^{1}\right)^{*}$ in the notations of $\left.\S 4.3\right)$; namely, this is true in the case where $\mathcal{M}$ classifies vector bundles over a scheme, local systems over a topological space, families curves or higher dimensional algebraic varieties, stable maps from a fixed scheme and so on. For some examples of the expected derived tangent spaces we refer again to [Ci-Ka1, Ci-Ka2].

Even if this does not determine exactly the derived extension, it certainly puts some constraints on the possible choices. To go a bit further, one notices that all the usual moduli functor occurring in algebraic geometry classify families of geometric objects over varying base schemes. To produce a derived extension $\mathbb{R} \mathcal{M}$, the main principle is then the following

Main principle: Let $\mathcal{M}$ be a moduli stack classifying certain kind of families of geometric objects over varying commutative algebras $A$. In order to guess what the extended moduli stack $\mathbb{R} \mathcal{M}$ should be, guess first what is a family of geometric objects of the same type parametrized by a commutative dga A.

In the case, for example, where $\mathcal{M}$ classifies families defined as maps with some properties (like for example in the case of the stack of curves), the derived analog is more or less clear. Anticipating a bit some definitions in the following sections, we say that our $D$-stacks will be simplicial presheaves on $D-A f f$ and that any cdga $A$ gives rise to a "representable" simplicial presheaf denoted by $\operatorname{Spec} A$ as well to its derived version (in the sense of model categories) $\mathbb{R} S p e c A$ (see Section 3.1). With these definitions at hand, one should say that a derived family over a cdga $A$ is just a map of simplicial presheaves $F \longrightarrow \mathbb{R}$ Spec $A$, having the same properties in the derived sense ${ }^{1}$. Then, a natural candidate for a derived family of the specific geometric objects classified by $\mathcal{M}$, is given by any such derived family that when restricted along Spec $H^{0}(A) \longrightarrow \operatorname{Spec} A$ it becomes equivalent to an object coming from $\mathcal{M}\left(\operatorname{Spec} H^{0}(A)\right)$ (note that $H^{0}(A)$ is a commutative ring and the latter Spec has the usual meaning). This condition, required in order to really get a derived extension, essentially says that the derived version of a family of geometric objects should reduce to a non-derived family of geometric objects in the non-derived or scheme-like direction. A typical example of this case is the one of $G$-torsors given in $\S 3.4$.

\section{The model category of $D$-stacks}

In this Section we will present the construction of a model category of D-stacks. It will be our derived version of the category of stacks that is commonly used in moduli theory, and all our examples of

\footnotetext{
${ }^{1}$ For example, as we will extend the notion of étale morphism of schemes to cdga's in $\S 2.2$, the same can be done with the notions of smooth, flat ... morphisms of schemes.
} 
derived moduli stacks will be objects of this category.

The main idea of the construction is the one used in [HAG-I], and consists of adopting systematically the functorial point of view. Schemes, or stacks, are sheaves over the category of commutative algebras. In the same way, $D$-stacks will be sheaf-like objects on the category of commutative differential graded algebras. This choice is in part justified also by the fact that all of the derived moduli spaces that we are aware of have reasonable models as objects in our category of $D$-stacks.

Before starting with the details of the construction, we would like to mention that $\mathrm{K}$. Behrend has independently used a similar approach to DAG that uses the 2-category of differential graded algebras (see [BeI, BeII]) (his approach is actually the 2-truncated version of ours). It is not clear to us that the constructions and results we are going to present in this work have reasonable analogs in his framework, as they use in an essential way higher homotopical informations that are partially lost when using any truncated version.

Conventions. For the sake of simplicity, we will work over the field of complex numbers $\mathbb{C}$. The expression cdga will always refer to a non-positively graded commutative differential graded algebra over $\mathbb{C}$ with differential of degree 1 . Therefore, a cdga $A$ looks like

$$
\cdots \longrightarrow A^{-n} \longrightarrow A^{-n+1} \longrightarrow \cdots \longrightarrow A^{-1} \longrightarrow A^{0} .
$$

The category CDGA of cdga's is endowed with its usual model category structure (see e.g. [Hin1]), for which fibrations (resp. equivalences) are epimorphisms in degree $\leq-1$ (resp. quasi-isomorphisms).

\section{$2.1 \quad D$-Pre-stacks}

We start by defining $D-A f f:=C D G A^{o p}$ to be the opposite category of cdga's, and we consider the category $S \operatorname{Pr}(D-A f f)$, of simplicial presheaves on $D-A f f$, or equivalently the category of functors from $C D G A$ to $S$ Set. The category $S \operatorname{Pr}(D-A f f)$ is endowed with its objectwise projective model structure in which fibrations and equivalences are defined objectwise (see [Hi, 13.10.17]).

For any cdga $A \in D-A f f$, we have the presheaf of sets represented by $A$, denoted by

$$
\begin{array}{clc}
h_{A}: D-A f f^{o p} & \longrightarrow & \operatorname{Set} \\
B & \mapsto & \operatorname{Hom}(B, A) .
\end{array}
$$

Considering a set as a constant simplicial set, we will look at $h_{A}$ as an object in $\operatorname{SPr}(D-A f f)$. The construction $A \mapsto h_{A}$ is clearly functorial in $A$, and therefore for any $u: A \rightarrow A^{\prime}$ in $D-A f f$, corresponding to a quasi-isomorphism of cdga's, we get a morphism $u: h_{A} \rightarrow h_{A^{\prime}}$ in $\operatorname{SPr}(D-A f f)$. These morphisms will simply be called quasi-isomorphisms.

Definition 2.1 The model category of D-pre-stacks is the left Bousfield localization of the model category $\operatorname{SPr}(D-A f f)$ with respect to the set of morphisms $\left\{u: h_{A} \rightarrow h_{A^{\prime}}\right\}$, where $u$ varies in the set of all quasi-isomorphisms. It will be denoted by $D-A f f^{\wedge}$.

Remark 2.2 1. The careful reader might object that the category $D-A f f$ and the set of all quasi-isomorphisms are not small, and therefore that Definition 2.1 does not make sense. If this happens (and only then), take two universes $\mathbb{U} \in \mathbb{V}$, define $C D G A$ as the category of $\mathbb{U}$ small cdga's and $S \operatorname{Pr}(D-A f f)$ as the category of functors from $C D G A$ to the category of $\mathbb{V}$-small simplicial sets. Definition 2.1 will now make sense. Alternatively, one could use suitable cardinality bounds.

2. In [HAG-I], the model category $D-A f f^{\wedge}$ was denoted by $(D-A f f, W)^{\wedge}$, where $W$ is the subcategory of quasi-isomorphisms. 
By general properties of left Bousfield localization (see [Hi]), the fibrant objects in $D-A f f^{\wedge}$ are the functors $F: C D G A \longrightarrow$ SSet satisfying the following two conditions

1. For any $A \in C D G A$, the simplicial set $F(A)$ is fibrant.

2. For any quasi-isomorphism $u: A \longrightarrow B$ in CDGA, the induced morphism $F(u): F(A) \longrightarrow F(B)$ is a weak equivalence of simplicial sets.

¿From this description, we conclude in particular, that the homotopy category $\operatorname{Ho}\left(D-A f f^{\wedge}\right)$ is naturally equivalent to the full sub-category of $\operatorname{Ho}(S \operatorname{Pr}(D-A f f))$ consisting of functors $F: C D G A \longrightarrow$ SSet sending quasi-isomorphisms to weak equivalences. We will use implicitly this description, and we will always consider $\operatorname{Ho}\left(D-A f f^{\wedge}\right)$ as embedded in $\operatorname{Ho}(\operatorname{SPr}(D-A f f))$.

Definition 2.3 Objects of $D-A f f^{\wedge}$ satisfying condition (2) above (i.e. sending quasi-isomorphisms to weak equivalences) will be called $D$-pre-stacks.

\section{$2.2 \quad D$-Stacks}

Now that we have constructed the model category of $D$-pre-stacks we will introduce some kind of étale topology on the category $D-A f f$. This will allow us to talk about a corresponding notion of étale local equivalences in $D-A f f^{\wedge}$, and to define the model category of $D$-stacks by including the local-to-global principle into the model structure.

We learned the following notion of formally étale morphism of cdga's from K. Behrend.

Definition 2.4 $A$ morphism $A \longrightarrow B$ in $C D G A$ is called formally étale if it satisfies the following two conditions.

1. The induced morphism $H^{0}(A) \longrightarrow H^{0}(B)$ is a formally étale morphism of commutative algebras.

2. For any $n<0$, the natural morphism of $H^{0}(B)$-modules

$$
H^{n}(A) \otimes_{H^{0}(A)} H^{0}(B) \longrightarrow H^{n}(B)
$$

is an isomorphism.

Remark 2.5 Though we did not check the details, it is probably true that a morphism $A \longrightarrow B$ of cdga's is formally étale in the sense of Definition 2.4 if and only if the relative cotangent complex $\mathbb{L} \Omega_{B / A}^{1}$, as defined in [Hin1], is acyclic. This would justify the chosen terminology.

¿From Definition 2.4 we now define the notion of étale covering families. For this, we recall that a morphism of cdga's $A \longrightarrow B$ is said to be finitely presented if $B$ is equivalent to a retract of a finite cell $A$-algebra (see for example [EKMM]). This is also equivalent to say that for any filtered systems $\left\{A \longrightarrow C_{i}\right\}_{i \in I}$, the natural morphism

$$
\operatorname{Colim}_{i \in I} \operatorname{Map}_{A / C D G A}\left(B, C_{i}\right) \longrightarrow \operatorname{Map}_{A / C D G A}\left(B, \operatorname{Colim}_{i \in I} C_{i}\right)
$$

is a weak equivalence (here $\operatorname{Map}_{A / C D G A}$ denotes the mapping spaces, or function complexes, of the model category $A / C D G A$ of cdga's under $A$, as defined in $[$ Ho, $\S 5.4])^{2}$.

\footnotetext{
${ }^{2}$ We warn the reader that if commutative algebras are considered as cdga's concentrated in degree zero, the notion of finitely presented morphisms of commutative algebras and the notion of finitely presented morphisms of cdga's are not the same. In fact, for a morphism of commutative algebras it is stronger to be finitely presented as a morphism of cdga's than as a morphism of algebras.
} 
Definition 2.6 A finite family of morphisms of cdga's

$$
\left\{A \longrightarrow B_{i}\right\}_{i \in I}
$$

is called an étale covering if it satisfies the following three conditions

1. For any $i \in I$, the morphism $A \longrightarrow B_{i}$ is finitely presented.

2. For any $i \in I$, the morphism $A \longrightarrow B_{i}$ is formally étale.

3. The induced family of morphisms of affine schemes

$$
\left\{\operatorname{Spec} H^{0}\left(B_{i}\right) \longrightarrow \operatorname{Spec} H^{0}(A)\right\}_{i \in I}
$$

is an étale covering.

The above definition almost defines a pre-topology on the category $D-A f f$. Indeed, stability and composition axioms for a pre-topology are satisfied, but the base change axiom is not. In general, the base change of an étale covering $\left\{A \longrightarrow B_{i}\right\}_{i \in I}$ along a morphism of $A \longrightarrow C$ will only be an étale covering if $A \longrightarrow C$ is a cofibration in CDGA. In other words, for the base change axiom to be satisfied one needs to replace fibered products by homotopy fibered products in $D-A f f$. Therefore, the étale covering families of Definition 2.6 do not satisfy the axioms for a pre-topology on $D-A f f$, but rather satisfy a homotopy analog of them. This is an example of a model pre-topology on the model category $D-A f f$, for which we refer the reader to [HAG-I, §4.3] where a precise definition is given.

In turns out that the data of a model pre-topology on a model category $M$ is more or less equivalent to the data of a Grothendieck topology on its homotopy category Ho( $M)$ (see [HAG-I, Prop. 4.3.5]). In our situation, the étale coverings of Definition 2.6 induce a Grothendieck topology, called the étale topology on the opposite of the homotopy category $\operatorname{Ho}(D-A f f)$ of cdga's. More concretely, a sieve $S$ over a cdga $A \in \operatorname{Ho}(D-A f f)$ is declared to be a covering sieve if it contains an étale covering family $\left\{A \longrightarrow B_{i}\right\}_{i \in I}$. The reader will check as an exercise that this defines a topology on $\operatorname{Ho}(D-A f f)$ (hint: one has to use that étale covering families are stable by homotopy pull-backs in $D-A f f$, or equivalently by homotopy push-outs in $C D G A)$. From now on, we will always consider $\operatorname{Ho}(D-A f f)$ as a Grothendieck site for this étale topology.

For a $D$-pre-stack $F \in D-A f f^{\wedge}$ (recall from Definition 2.3 that this implies that $F$ sends quasiisomorphisms to weak equivalences), we define its presheaf of connected components

$$
\begin{aligned}
& \pi_{0}^{p r}(F): \quad D-A f f^{o p} \longrightarrow \quad \text { Set } \\
& A \quad \mapsto \quad \pi_{0}(F(A)) .
\end{aligned}
$$

As the object $F$ is a $D$-pre-stack (see 2.3 ), the functor $\pi_{0}^{p r}(F)$ will factors through the homotopy category

$$
\begin{aligned}
& \pi_{0}^{p r}(F): \operatorname{Ho}(D-A f f)^{o p} \quad \longrightarrow \quad S e t \\
& A \quad \mapsto \quad \pi_{0}(F(A)) \text {. }
\end{aligned}
$$

We can consider the sheaf $\pi_{0}(F)$ associated to the presheaf $\pi_{0}^{p r}$ in the étale topology on $\operatorname{Ho}(D-A f f)$. The sheaf $\pi_{0}(F)$ is called the 0-homotopy sheaf of the $D$-pre-stack $F$. Now, if $F \in D-A f f^{\wedge}$ is any simplicial presheaf, then one can apply the above construction to one of its fibrant models $R F$. This allows us to define its 0 -th homotopy sheaf as $\pi_{0}(F):=\pi_{0}(R F)$.

As for the case of simplicial presheaves (see [Ja1]), one can also define higher homotopy sheaves, which are sheaves of groups and abelian groups on the sites $\operatorname{Ho}(D-A f f / A)$ for various cdga's $A$. 
Precisely, let $F$ be a $D$-pre-stacks and $s \in F(A)_{0}$ a point over a cdga $A \in D-A f f$. We define the $n$-th homotopy group presheaf pointed at $s$ by

$$
\begin{array}{cccc}
\pi_{n}^{p r}(F, s): & D-A f f^{o p} / A=A / C D G A & \longrightarrow & G p \\
(u: A \rightarrow B) & \mapsto & \pi_{n}\left(F(B), u^{*}(s)\right) .
\end{array}
$$

Again, as $F$ is a $D$-pre-stack, these presheaves factor through the homotopy category

$$
\begin{array}{ccc}
\pi_{n}^{p r}(F, s): \operatorname{Ho}\left(D-A f f^{o p} / A\right)=\operatorname{Ho}(A / C D G A) & \longrightarrow & G p \\
(u: A \rightarrow B) & \mapsto & \pi_{n}\left(F(B), u^{*}(s)\right) .
\end{array}
$$

The étale model pre-topology on $D-A f f$ also induces Grothendieck topologies on the various homotopy categories $\operatorname{Ho}(A / C D G A)$, and therefore one can consider the sheaves associated to $\pi_{n}^{p r}(F, s)$. These sheaves are called the $n$-th homotopy sheaves of $F$ pointed at $s$ and are denoted by $\pi_{n}(F, s)$. As before, if $F$ is any object in $D-A f f^{\wedge}$, one can define $\pi_{n}(F, s):=\pi_{n}(R F, s)$ for $R F$ a fibrant replacement of $F$.

The notion of homotopy sheaves defined above gives rise to the following notion of local equivalences.

Definition 2.7 A morphism $f: F \longrightarrow F^{\prime}$ in $D-A f f^{\wedge}$ is called a (étale) local equivalence if it satisfies the following two conditions

1. The induced morphism of sheaves $\pi_{0}(F) \longrightarrow \pi_{0}\left(F^{\prime}\right)$ is an isomorphism.

2. For any $A \in D-A f f$, and any point $s \in F(A)$, the induced morphism of sheaves $\pi_{n}(F, s) \longrightarrow$ $\pi_{n}\left(F^{\prime}, f(s)\right)$ is an isomorphism.

One of the key results of "HAG" is the following theorem. It is a very special case of the existence theorem [HAG-I, §4.6], which extends the existence of the local model structure on simplicial presheaves (see [Ja1]) to the case of model sites.

Theorem 2.8 There exists a model category structure on $D-A f f^{\wedge}$ for which the equivalences are the local equivalences and the cofibrations are the cofibrations in the model category $D-A f f^{\wedge}$ of D-pre-stacks.

This model category is called the model category of $D$-stacks for the étale topology, and is denoted by $D-A f f^{\sim}$.

The reason for calling $D-A f f^{\sim}$ the model category of $D$-stacks is the following proposition. It follows from [HAG-I, 4.6.3], which is a generalization to model sites of the main theorem of [DHI].

Proposition 2.9 An object $F \in D-A f f^{\sim}$ is fibrant if and only if it satisfies the following three conditions

1. For any $A \in D-A f f$, the simplicial set $F(A)$ is fibrant.

2. For any quasi-isomorphism of cdga's $A \longrightarrow B$, the induced morphism $F(A) \longrightarrow F(B)$ is a weak equivalence.

3. For any cdga $A$, and any étale hyper-cover in D-Aff (see [HAG-I] for details) $A \longrightarrow B_{*}$, the induced morphism

$$
F(A) \longrightarrow \operatorname{Holim}_{n \in \Delta} F\left(B_{n}\right)
$$

is a weak equivalence. 
Condition (3) is called the stack condition for the étale topology. Note that a typical étale hypercover of cdga's $A \longrightarrow B_{*}$ is given by the homotopy co-nerve of an étale covering morphism $A \longrightarrow B$

$$
B_{n}:=\underbrace{B \otimes_{A}^{\mathbb{L}} B \otimes_{A}^{\mathbb{L}} \cdots \otimes_{A}^{\mathbb{L}} B}_{n \text { times }} .
$$

Condition (3) for these kind of hyper-covers is the most commonly used descent condition, but as first shown in $[\mathrm{DHI}]$ requiring descent with respect to all étale hyper-coverings is necessary for Proposition 2.9 to be correct.

Definition 2.10 $A$ D-stack is any object $F \in D-A f f^{\sim}$ satisfying conditions (2) and (3) of Proposition 2.9. By abuse of language, objects in the homotopy category $\operatorname{Ho}\left(D-A f f^{\sim}\right)$ will also be called $D$-stacks. A morphism of D-stacks is a morphism in the homotopy category $\operatorname{Ho}\left(D-A f f^{\sim}\right)$.

The second part of the definition is justified because the homotopy category $\operatorname{Ho}\left(D-A f f^{\sim}\right)$ is naturally equivalent to the full sub-category of $\operatorname{Ho}(S \operatorname{Pr}(D-A f f))$ consisting of objects satisfying conditions (2) and (3) of Proposition 2.9.

\subsection{Operations on $D$-stacks}

One of the main consequences of the existence of the model structure on $D-A f f^{\sim}$ is the possibility to define several standard operations on $D$-stacks, analogous to the ones used in sheaf theory (limits, colimits, sheaves of morphisms ...).

First of all, the category $D-A f f^{\sim}$ being a category of simplicial presheaves, it comes with a natural enrichment over the category of simplicial sets. This makes $D-A f f^{\sim}$ into a simplicial model category (see [Ho, 4.2.18]). In particular, one can define in a standard way the derived simplicial Hom's (well defined in the homotopy category $\operatorname{Ho}(S S e t))$,

$$
\mathbb{R} \underline{\operatorname{Hom}}(F, G):=\underline{\operatorname{Hom}}(Q F, R G),
$$

where $Q$ is a cofibrant replacement functor, $R$ is a fibrant replacement functor, and $\underline{H o m}$ are the simplicial Hom's sets of $D-A f f^{\sim}$. These derived simplicial Hom's allows one to consider spaces of morphisms between $D$-stacks, in the same way as one commonly considers groupoids of morphisms between stacks in groupoids (see [La-Mo]).

This simplicial structure also allows one to define exponentials by simplicial sets. For an object $F \in D-A f f^{\sim}$ and $K \in S S e t$, one has a well defined object in $\operatorname{Ho}\left(D-A f f^{\sim}\right)$

$$
F^{\mathbb{R} K}:=(R F)^{K}
$$

which satisfies the usual adjunction formula

$$
\mathbb{R} \underline{\operatorname{Hom}}\left(G, F^{\mathbb{R} K}\right) \simeq \mathbb{R} \underline{\operatorname{Hom}}(K, \underline{\mathbb{H o m}}(G, F)) .
$$

The existence of the model structure $D-A f f^{\sim}$ also implies the existence of homotopy limits and homotopy colimits, as defined in [Hi, §19]. The existence of these homotopy limits and colimits is the analog of the fact that category of sheaves have all kind of limits and colimits. We will use in particular homotopy pull-backs i.e. homotopy limits of diagrams $F \ll H \longrightarrow G$, that will be denoted by

$$
F \times{ }_{H}^{h} G:=\operatorname{Holim}\{F \longleftarrow H \longrightarrow G\} .
$$

Finally, one can show that the homotopy category $\operatorname{Ho}\left(D-A f f^{\sim}\right)$ is cartesian closed (see [HAG-I, $\S 4.7])$. Therefore, for any two object $F$ and $G$, there exists an object $\mathbb{R} \mathcal{H O} \mathcal{M}(F, G) \in \operatorname{Ho}\left(D-A f f^{\sim}\right)$, which is determined by the natural isomorphisms

$$
\underline{\mathbb{H} \operatorname{Hom}}(F \times G, H) \simeq \underline{\mathbb{H} \underline{\operatorname{Hom}}}(F, \mathbb{R} \mathcal{H O} \mathcal{M}(G, H)) .
$$


We say that $\mathbb{R} \mathcal{H} \mathcal{O} \mathcal{M}(F, G)$ is the $D$-stack of morphisms from $F$ to $G$, analogous to the sheaf of morphisms between two sheaves.

If one looks at these various constructions, one realizes that $D-A f f^{\sim}$ has all the homotopy analogs of the properties that characterize Grothendieck topoi. To be more precise, C. Rezk has defined a notion of homotopy topos (we rather prefer the expression model topos), which are model categories behaving homotopically very much like a usual topos. The standard examples of such homotopy topoi are model categories of simplicial presheaves on some Grothendieck site, but not all of them are of this kind; the model category $D-A f f^{\sim}$ is in fact an example of a model topos which is not equivalent to model categories of simplicial presheaves on some site (see [HAG-I, §3.8] for more details on the subject).

\section{$3 \quad$ First examples of $D$-stacks}

Before going further with the geometric properties of $D$-stacks, we would like to present some examples. More examples will be given in the Section 5 .

\subsection{Representables}

The very first examples of schemes are affine schemes. In the same way, our first example of $D$-stacks are representable D-stacks ${ }^{3}$.

We start by fixing a fibrant resolution functor $\Gamma$ on the model category $C D G A$. Recall that this means that for any cdga $B, \Gamma(B)$ is a simplicial object in $C D G A$, together with a natural morphism $B \longrightarrow \Gamma(B)$ that makes it into a fibrant replacement for the Reedy model structure on simplicial objects (see [Ho, §5.2]). In the present situation, one could choose the following standard fibrant resolution functor

$$
\begin{aligned}
& \Gamma(B): \Delta^{o p} \longrightarrow \quad C D G A \\
& {[n] \quad \mapsto \quad \Gamma(B)_{n}:=B \otimes \Omega_{\Delta^{n}}^{*} .}
\end{aligned}
$$

Here $\Omega_{\Delta^{n}}^{*}$ is the cdga (exceptionally positively graded) of algebraic differential forms on the standard algebraic $n$-simplex. Of course the cdga $B \otimes \Omega_{\Delta^{n}}^{*}$ is not non-positively graded, but one can always take its truncation in order to see it as an object in $C D G A$.

Now, for any cdga $A$, we define a functor

$$
\begin{array}{ccc}
\text { Spec } A: C D G A & \longrightarrow & \text { SSet } \\
B & \mapsto & \operatorname{Hom}_{C D G A}(A, \Gamma(B)),
\end{array}
$$

that is considered as an object in $D-A f f^{\sim}$. This construction is clearly functorial in $A$ and gives rise to a functor

$$
\text { Spec }: C D G A^{o p}=D-A f f \longrightarrow D-A f f^{\sim} .
$$

The functor Spec is almost a right Quillen functor: it preserves fibrations, trivial fibrations and limits, but does not have a left adjoint. However, it has a well defined right derived functor

$$
\mathbb{R} \text { Spec }: \operatorname{Ho}(C D G A)^{o p}=\operatorname{Ho}(D-A f f) \longrightarrow \operatorname{Ho}\left(D-A f f^{\sim}\right) .
$$

A fundamental property of this functor is the following lemma.

Lemma 3.1 The functor $\mathbb{R} S p e c$ is fully faithful. More generally, for two cdga's $A$ and $B$, it induces a natural equivalence on the mapping spaces

$$
\mathbb{R} \underline{\operatorname{Hom}}(A, B) \simeq \mathbb{R} \underline{\operatorname{Hom}}(\mathbb{R} \operatorname{Spec} B, \mathbb{R} S \text { pec } A) .
$$

\footnotetext{
${ }^{3}$ We could as well have called them affine $D$-stacks.
} 
The above lemma contains two separated parts. The first part states that $\mathbb{R} S p e c$ is fully faithful when considered to have values in $\operatorname{Ho}\left(D-A f f^{\wedge}\right)$ (i.e. when one forgets about the topology). This first part is a very general result that we call Yoneda lemma for model categories (see [HAG-I, §4.2]). The second part of the lemma states that for a cofibrant cdga $A$, the object $\operatorname{Spec}(A)$ is a $D$-stack (see Definition 2.10). This is not a general fact, and of course depends on the choice of the topology. Another way to express this last result is to say that the étale topology is sub-canonical.

Definition 3.2 A D-stack isomorphic in $\operatorname{Ho}\left(D-A f f^{\sim}\right)$ to some $\mathbb{R} S p e c A$ is called a representable D-stack.

In particular, Lemma 3.1 implies that the full subcategory of $\operatorname{Ho}(D-A f f)^{\sim}$ consisting of representable $D$-stacks is equivalent to the homotopy category of cdga's.

\subsection{Stacks vs. $D$-stacks}

Our second example of $D$-stacks are simply stacks. In other words, any stack defined over the category of affine schemes with the étale topology gives rise to a $D$-stack.

Let $A l g$ be the category of commutative $\mathbb{C}$-algebras, and $A f f=A l g^{o p}$ its opposite category. Recall that there exists a model category of simplicial presheaves on $A f f$ for the étale topology (see [Ja1]). We will consider its projective version described in [Bl], and denote it by $A f f^{\sim}$. This model category is called the model category of stacks for the étale topology. Its homotopy category $\operatorname{Ho}\left(A f f^{\sim}\right)$ contains as full subcategories the category of sheaves of sets and the category of stacks in groupoids (see e.g. [La-Mo]). More generally, one can show that the full subcategory of $n$-truncated objects in $\operatorname{Ho}\left(A f f^{\sim}\right)$ is naturally equivalent to the homotopy category of stacks in $n$-groupoids (unfortunately there are no references for this last result until now but the reader might consult [Hol] for the case $n=1$ ). In particular, $\operatorname{Ho}\left(A f f^{\sim}\right)$ contains as a full subcategory the category of schemes, and more generally of Artin stacks.

There exists an adjunction

$$
H^{0}: C D G A \longrightarrow A l g \quad C D G A \longleftarrow A l g: j,
$$

for which $j$ is the full embedding of $A l g$ in $C D G A$ that sends a commutative algebra to the corresponding cdga concentrated in degree 0. Furthermore, this adjunction is a Quillen adjunction when $A l g$ is endowed with its trivial model structure (as written above, $j$ is on the right and $H^{0}$ is its left adjoint). This adjunction induces various adjunctions between the category of simplicial presheaves

$$
\begin{gathered}
j !: A f f^{\sim} \longrightarrow D-A f f^{\sim} \quad A f f^{\sim} \longleftarrow D-A f f^{\sim}: j^{*} \\
j^{*}: D-A f f^{\sim} \longrightarrow A f f^{\sim} \quad D-A f f^{\sim} \longleftarrow A f f^{\sim}:\left(H^{0}\right)^{*}
\end{gathered}
$$

One can check that these adjunction are Quillen adjunction (where the functors written on the left are left Quillen). In particular we conclude that $j^{*}$ is right and left Quillen, and therefore preserves equivalences. ¿From this we deduce easily the following important fact.

Lemma 3.3 The functor

$$
i:=\mathbb{L} j_{!}: \operatorname{Ho}\left(A f f^{\sim}\right) \longrightarrow \operatorname{Ho}\left(D-A f f^{\sim}\right)
$$

is fully faithful.

The important consequence of the previous lemma is that $\operatorname{Ho}\left(D-A f f^{\sim}\right)$ contains schemes, algebraic stacks ..., as full sub-categories. 
Warning: The full embedding $i$ does not commute with homotopy pull-backs, nor with internal Hom- $D$-stacks.

This warning is the real heart of DAG: the category of $D$-stacks contains usual stacks, but these are not stable under the standard operations of $D$-stacks. In other words, if one starts with some schemes and performs some constructions on these schemes, considered as $D$-stacks, the result might not be a scheme anymore. This is the main reason why derived moduli spaces are not schemes, or stacks in general!

Notations. In order to avoid confusion, a scheme or a stack $X$, when considered as a $D$-stack will always be denoted by $i(X)$, or simply by $i X$.

The full embedding $i=\mathbb{L} j$ ! has a right adjoint $\mathbb{R} j^{*}=j^{*}$. It will be denoted by

$$
h^{0}:=j^{*}: \operatorname{Ho}\left(D-A f f^{\sim}\right) \longrightarrow \operatorname{Ho}\left(A f f^{\sim}\right),
$$

and called the truncation functor. Note that for any cdga, one has

$$
h^{0}(\mathbb{R} S \operatorname{Spec} A) \simeq \operatorname{Spec} H^{0}(A)
$$

which justifies the notation $h^{0}$. Note also that for any $D$-stack $F$, and any commutative algebra $A$, one has

$$
F(A) \simeq \mathbb{R} \underline{\operatorname{Hom}}(i \operatorname{Spec} A, F) \simeq \mathbb{R} \underline{\operatorname{Hom}}\left(\operatorname{Spec} A, h^{0}(F)\right) \simeq h^{0}(F)(A) .
$$

This shows that a $D$-stack $F$ and its truncation $h^{0}(F)$ have the same points with values in commutative algebras. Of course, $F$ and $h^{0}(F)$ do not have the same points with values in cdga's in general, except when $F$ is of the form $i F^{\prime}$ for some stack $F^{\prime} \in \operatorname{Ho}\left(A f f^{\sim}\right)$.

Terminology. Points with values in commutative algebras will be called classical points.

We just saw that a $D$-stack $F$ and its truncation $h^{0}(F)$ always have the same classical points.

Given two stacks $F$ and $G$ in $A f f^{\sim}$, there exists a stack of morphisms $\mathbb{R} \mathcal{H O} \mathcal{M}(F, G)$, that is the derived internal Hom's of the model category $A f f^{\sim}$ (see [HAG-I, §4.7]). As remarked above, the two objects $i \mathbb{R} \mathcal{H O} \mathcal{M}(F, G)$ and $\mathbb{R} \mathcal{H O} \mathcal{M}(i F, i G)$ are different in general. However, one has

$$
h^{0}(\mathbb{R} \mathcal{H O} \mathcal{M}(i F, i G)) \simeq \mathbb{R} \mathcal{H O} \mathcal{M}(F, G),
$$

showing that $i \mathbb{R} \mathcal{H O M}(F, G)$ and $\mathbb{R} \mathcal{H O} \mathcal{M}(i F, i G)$ have the same classical points.

\section{3 dg-Schemes}

We have just seen that the homotopy category of $D$-stacks $\operatorname{Ho}\left(D-A f f^{\sim}\right)$ contains the categories of schemes and algebraic stacks. We will now relate the notion of dg-schemes of [Ci-Ka1, Ci-Ka2] to $D$-stacks.

Recall that a dg-scheme is a pair $\left(X, \mathcal{A}_{X}\right)$, consisting of a scheme $X$ and a sheaf of $\mathcal{O}_{X}$-cdga's on $X$ such that $\mathcal{A}_{X}^{0}=\mathcal{O}_{X}$ (however, this last condition does not seem so crucial). For the sake of simplicity we will assume that $X$ is quasi-compact and separated. We can therefore take a finite affine open covering $\mathcal{U}=\left\{U_{i}\right\}_{i}$ of $X$, and consider its nerve $N(\mathcal{U})$ (which is a simplicial scheme)

$$
\begin{aligned}
N(\mathcal{U}): & \Delta^{o p} \\
{[n] } & \mapsto \quad \coprod_{i_{0}, \ldots, i_{n}} U_{i_{0}, \ldots, i_{n}}
\end{aligned}
$$


where, as usual, $U_{i_{0}, \ldots, i_{n}}=U_{i_{0}} \cap U_{i_{1}} \cap \ldots U_{i_{n}}$. Note that as $X$ is separated and the covering is finite, $N(\mathcal{U})$ is in fact a simplicial affine scheme.

For each integer $n$, let $A(n)$ be the cdga of global sections of $\mathcal{A}_{X}$ on the scheme $N(\mathcal{U})_{n}$. In other words, one has

$$
A(n)=\prod_{i_{0}, \ldots, i_{n}} \mathcal{A}_{X}\left(U_{i_{0}, \ldots, i_{n}}\right) .
$$

The simplicial structure on $N(\mathcal{U})$ makes $[n] \mapsto A(n)$ into a co-simplicial diagram of cdga's. By applying levelwise the functor $\mathbb{R} S p e c$, we get a simplicial object $[n] \mapsto \mathbb{R} S p e c A(n)$ in $D-A f f^{\sim}$. We define the $D$-stack $\Theta\left(X, \mathcal{A}_{X}\right) \in \operatorname{Ho}\left(D-A f f^{\sim}\right)$ to be the homotopy colimit of this diagram

$$
\Theta\left(X, \mathcal{A}_{X}\right):=\text { Hocolim }_{[n] \in \Delta^{o p}} \mathbb{R} S \operatorname{Spec} A(n) .
$$

If we denote by $\mathrm{Ho}(d g-S c h)$ the category of dg-schemes localized along quasi-isomorphisms ([Ci-Ka1] $)$, one can check, that $\left(X, \mathcal{A}_{X}\right) \mapsto \Theta\left(X, \mathcal{A}_{X}\right)$ defines a functor

$$
\Theta: \operatorname{Ho}(d g-S c h) \longrightarrow \operatorname{Ho}\left(D-A f f^{\sim}\right),
$$

from the homotopy category of (quasi-compact and separated) dg-schemes to the homotopy category of $D$-stacks. This functor allows us to consider dg-schemes as $D$-stacks.

\section{Question: Is the functor $\Theta$ fully faithful?}

We do not know the answer to this question, and there are no real reasons for this answer to be positive. As already explained in the Introduction, the difficulty comes from the fact that the homotopy category of dg-schemes seems quite difficult to describe. In a way, it might not be so important to know the answer to the above question, as until now morphisms in the homotopy category of dg-schemes have never been taken into account seriously, and only the objects of the category $\operatorname{Ho}(d g-S c h)$ have been shown to be relevant. More fundamental is the existence of the functor $\Theta$ which allows to see the various dg-schemes constructed in [Ka2, Ci-Ka1, Ci-Ka2] as objects in $\operatorname{Ho}\left(D-A f f^{\sim}\right)$.

Remark 3.4 The above construction of $\Theta$ can be extended from dg-schemes to (Artin) dg-stacks.

\subsection{The $D$-stack of $G$-torsors}

As our last example, we present the $D$-stack of $G$-torsors where $G$ is a linear algebraic group $G$. As an object in $\operatorname{Ho}\left(D-A f f^{\sim}\right)$ it is simply $i B G$ (where $B G$ is the usual stack of $G$-torsors), but we would like to describe explicitly the functor $C D G A \longrightarrow S S e t$ it represents.

Let $H:=\mathcal{O}(G)$ be the Hopf algebra associated to $G$. By considering it as an object in the model category of commutative differential graded Hopf algebras, we can take a cofibrant model $Q H$ of $H$, as a dg-Hopf algebra. It is not very hard to check that $Q H$ is also a cofibrant model for $H$ in the model category of cdga's. Using the co-algebra structure on $Q H$, one sees that the simplicial presheaf

$$
\operatorname{Spec} Q H: D-A f f^{o p} \longrightarrow S S e t
$$

has a natural structure of group-like object. In other words, $S p e c Q H$ is a presheaf of simplicial groups on $D-A f f$. As the underlying simplicial presheaf of $\operatorname{Spec} Q H$ is naturally equivalent to $\mathbb{R} S$ pec $H \simeq i G$, we will simply denote this presheaf of simplicial groups by $i G$.

Next, we consider the category $i G-M o d$, of objects in $D-A f f^{\sim}$ together with an action of $i G$. If one sees $i G$ as a monoid in $D-A f f^{\sim}$, the category $i G-M o d$ is simply the category of modules over $i G$. The category $i G-M o d$ is equipped with a notion of weak equivalences, that are 
defined through the forgetful functor $i G-\operatorname{Mod} \longrightarrow D-A f f^{\sim}$ (therefore a morphism of $i G$-modules is a weak equivalence if the morphism induced on the underlying objects is a weak equivalence in $\left.D-A f f^{\sim}\right)$. More generally, there is a model category structure on $i G-\operatorname{Mod}$, such that fibrations and equivalences are defined on the underlying objects. For any object $F \in i G-M o d$, we also get an induced model structure on the comma category $i G-M o d / F$. In particular, it makes sense to say that two objects $G \longrightarrow F$ and $G^{\prime} \longrightarrow F$ in $i G-M o d$ are equivalent over $F$, if the corresponding objects in $\operatorname{Ho}(i G-M o d / F)$ are isomorphic.

Let $Q$ be a cofibrant replacement functor in the model category $C D G A$. For any cdga $A$, we have Spec $Q A \in D-A f f^{\sim}$, the representable $D$-stack represented by $A \in D-A f f$, that we will consider as $i G$-module for the trivial action. A $G$-torsor over $A$ is defined to be a $i G$-module $F \in i G-M o d$, together with a fibration of $i G$-modules $F \longrightarrow \operatorname{Spec} Q A$, such that there exists an étale covering $A \longrightarrow B$ with the property that the object

$$
F \times{ }_{\operatorname{Spec} Q A} \operatorname{Spec} Q B \longrightarrow \operatorname{Spec} Q B
$$

is equivalent over $\operatorname{Spec} Q B$ to $i G \times \operatorname{Spec} Q B \longrightarrow \operatorname{Spec} Q B$ (where $i G$ acts on itself by left translations).

For a cdga $A, G$-torsors over $A$ form a full sub-category of $i G-\operatorname{Mod} / \operatorname{Spec} Q A$, that will be denoted by $G-\operatorname{Tor} s(A)$. This category has an obvious induced notion of weak equivalences, and these equivalences form a subcategory denoted by $w G-\operatorname{Tor} s(A)$. Transition morphisms $w G-\operatorname{Tors}(A) \longrightarrow$ $w G-\operatorname{Tors}(B)$ can be defined for any morphism $A \longrightarrow B$ by sending a $G$-torsor $F \longrightarrow S p e c Q A$ to the pull-back $F \times_{\operatorname{Spec} Q A} \operatorname{Spec} Q B \longrightarrow \operatorname{Spec} Q B$. With a bit of care, one can make this construction into a (strict) functor

$$
\begin{array}{ccc}
C D G A & \longrightarrow & \operatorname{Cat} \\
A & \mapsto & w G-\operatorname{Tors}(A) .
\end{array}
$$

We are now ready to define our functor $\mathbb{R} B G: C D G A \longrightarrow S$ Set by $A \mapsto|w G-\operatorname{Tor} s(A)|$, where $|w G-\operatorname{Tors}(A)|$ is the nerve of the category $w G-\operatorname{Tor} s(A)$. The following result says that $\mathbb{R} B G$ is the associated D-stack to $i B G$ (recall that $B G$ is the Artin stack of $G$-torsors, and that $i B G$ is its associated $D$-stack defined through the embedding $i$ of Lemma 3.3).

Proposition 3.5 1. The object $\mathbb{R} B G \in D-A f f^{\sim}$ is a D-stack.

2. There exists an isomorphism $i B G \simeq \mathbb{R} B G$ in the homotopy category $\operatorname{Ho}\left(D-A f f^{\sim}\right)$.

An important case is $G=G l_{n}$, for which we get that the image under $i$ of the stack $V e c t_{n}$ of vector bundles of rank $n$ is equivalent to $\mathbb{R} B G l_{n}$ as defined above.

\section{The geometry of $D$-stacks}

We are now ready to start our geometric study of $D$-stacks. We will define in this Section a notion of (1)-geometric $D$-stack, analogous to the notion of algebraic stack (in the sense of Artin). We will also present the theory of tangent D-stacks, as well as its relations to the cotangent complex.

\subsection{Geometricity}

A 1-geometric D-stack is a quotient of a disjoint union of representable $D$-stacks by the action of a smooth affine groupoid. In order to define precisely this notion, we need some preliminaries.

1. Let $f: F \longrightarrow F^{\prime}$ be a morphism in $\operatorname{Ho}\left(D-A f f^{\sim}\right)$. We say that $f$ is a representable morphism, if for any cdga $A$, and any morphism $\mathbb{R} S$ pec $A \longrightarrow F^{\prime}$, the homotopy pull-back $F \times{ }_{F^{\prime}}^{h} \mathbb{R} S$ pec $A$ is a representable $D$-stack (see Definition 3.2). 
2. We say that a $D$-stack $F$ has a representable diagonal if the diagonal morphism $\Delta: F \longrightarrow F \times F$ is representable. Equivalently, $F$ has a representable diagonal if any morphism $\mathbb{R} S p e c A \longrightarrow F$ from a representable $D$-stack is a representable morphism.

3. Let $u: A \longrightarrow B$ be a morphism of cdga's. We say that $u$ is strongly smooth ${ }^{4}$ if there exists an étale covering $B \longrightarrow B^{\prime}$, and a factorization

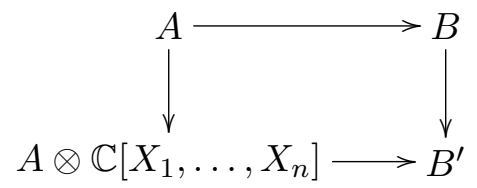

with $A \otimes \mathbb{C}\left[X_{1}, \ldots, X_{n}\right] \longrightarrow B^{\prime}$ formally étale; here $\mathbb{C}\left[X_{1}, \ldots, X_{n}\right]$ is the usual polynomial ring, viewed as a cdga concentrated in degree zero. This is an extension of one of the many equivalent characterizations of smoothness for morphisms of schemes (see [Mil, Prop. 3.24 (b)]); we learn it from $[\mathrm{MCM}]$ in which smooth morphisms (called there thh-smooth) between $\mathbb{S}$-algebras are defined.

4. A representable morphism of $D$-stacks $f: F \longrightarrow F^{\prime}$ is called strongly smooth, if for any morphism from a representable $D$-stack $\mathbb{R} S$ pec $A \longrightarrow F^{\prime}$, the induced morphism

$$
F \times{ }_{F^{\prime}}^{h} \mathbb{R} \text { Spec } A \longrightarrow \mathbb{R} \text { Spec } A
$$

is induced by a strongly smooth morphism of cdga's.

5. A morphism $f: F \longrightarrow F^{\prime}$ in $\operatorname{Ho}\left(D-A f f^{\sim}\right)$ is called a covering (or an epimorphism), if the induced morphism $\pi_{0}(F) \longrightarrow \pi_{0}\left(F^{\prime}\right)$ is an epimorphism of sheaves.

Note that definition (4) above makes sense because of (1) and because the functor $A \mapsto \mathbb{R} S p e c A$ is fully faithful on the homotopy categories.

Using these notions, we give the following

Definition 4.1 A D-stack $F$ is strongly 1-geometric (or simply, strongly geometric) if it satisfies the following two conditions

1. F has a representable diagonal.

2. There exist representable D-stacks $\mathbb{R} S p e c A_{i}$, and a covering

$$
\coprod_{i} \mathbb{R} \operatorname{Spec} A_{i} \longrightarrow F,
$$

such that each of the morphisms $\mathbb{R}$ Spec $A_{i} \longrightarrow F$ (which is representable by 1.) is strongly smooth. Such a family of morphisms will be called a strongly smooth atlas of $F$.

Remark 4.2 Objects satisfying Definition 4.1 are called strongly 1-geometric $D$-stacks as there exists a more general notion of strongly $n$-geometric $D$-stacks, obtained by induction as suggested in [S1]. The notion of strongly 1-geometric $D$-stacks will be enough for our purposes (except for our last example in section 5), and we will simply use the expression strongly geometric D-stacks.

The following proposition collects some of the basic properties of strongly geometric $D$-stacks.

\footnotetext{
${ }^{4}$ The expression smooth morphism will be used for a weaker notion in $\S 4.4$.
} 


\section{Proposition 4.3 1. Representable D-stacks are strongly geometric.}

2. The homotopy pull-back of a diagram of strongly geometric D-stacks is again a strongly geometric $D$-stack. In particular strongly geometric D-stacks are stable by finite homotopy limits.

3. If $F$ is any algebraic stack (in the sense of Artin, see [La-Mo]) with an affine diagonal, then iF is a strongly geometric D-stack.

4. If $F$ is a strongly geometric D-stack then $h^{0}(F)$ is an algebraic stack (in the sense of Artin) with affine diagonal. In particular, $i h^{0}(F)$ is again a strongly geometric D-stack.

5. For any dg-scheme $\left(X, \mathcal{A}_{X}\right),\left(X\right.$ separated and quasi-compact), $\Theta\left(X, \mathcal{A}_{X}\right)$ (see $\left.\S 3.3\right)$ is a strongly geometric D-stack.

In particular, Proposition 3.5 and point (3) above, tell us that the derived stack $\mathbb{R} B G$ of $G$-torsors is a strongly geometric $D$-stack for any linear algebraic group $G$.

We are not going to present the theory in details in this work, but we would like to mention that standard notions in algebraic geometry (e.g. smooth or flat morphisms, sheaves, cohomology ...) can be extended to strongly geometric $D$ stacks. We refer to [La-Mo] and [S1] for the main outline of the constructions. The reader will find all details in [HAG-II].

\subsection{Modules, linear $D$-stacks and $K$-theory}

Let $\mathbb{G}_{a}$ be the additive group scheme (over $\mathbb{C}$ ) and consider the object $i \mathbb{G}_{a} \in \operatorname{Ho}\left(D-A f f^{\sim}\right.$ ). It has a nice model in $D-A f f^{\sim}$ which is $\operatorname{Spec} \mathbb{C}[T]$ that we will denote by $\mathcal{O}$ (note that $\mathbb{C}[T]$ as a cdga in degree 0 is a cofibrant object). The $D$-stack $\mathcal{O}$ is actually an object in commutative $\mathbb{C}$-algebras, explicitly given by

$$
\begin{aligned}
& \mathcal{O}: C D G A \longrightarrow(\mathbb{C}-A l g)^{\Delta^{o p}} \\
& A \quad \mapsto \quad\left([n] \mapsto \Gamma_{n}(A)^{0}\right),
\end{aligned}
$$

where $\Gamma$ is a fibrant resolution functor. The $D$-stack $\mathcal{O}$ is called the structural $D$-stack.

Let us now fix a $D$-stack $F$, and consider the comma category $D-A f f^{\sim} / F$ of $D$-stacks over $F$; this category is again a model category for the obvious model structure. We define the relative structural D-stack by

$$
\mathcal{O}_{F}:=(\mathcal{O} \times F \longrightarrow F) \in D-A f f^{\sim} / F
$$

Since $\mathcal{O}$ is a $\mathbb{C}$-algebra object, we deduce immediately that $\mathcal{O}_{F}$ is also a $\mathbb{C}$-algebra object in the comma model category $D-A f f^{\sim} / F$.

Then we can consider the category $\mathcal{O}_{F}-M o d$, of objects in $\mathcal{O}_{F}$-modules in the category $D-$ $A f f^{\sim} / F$. If one defines equivalences and fibrations through the forgetful functor $D-A f f^{\sim} / F \longrightarrow$ $D-A f f^{\sim}$, the category $\mathcal{O}_{F}-\operatorname{Mod}$ becomes a model category. It has moreover a natural tensor product structure $\otimes_{\mathcal{O}_{F}}$. The model category $\mathcal{O}_{F}-$ Mod is called the model category of $\mathcal{O}$-modules on $F$.

Let $A$ be a cdga and $M$ be an (unbounded) $A$-dg module. We define a $\mathcal{O}_{\text {Spec }} A_{\text {-module }} \widetilde{M}$ in the following way.

Let $\Gamma$ be a fibrant resolution functor on the model category $C D G A$. For any cdga $B$, and any integer $n$, we define $\widetilde{M}(B)_{n}$ as the set of pairs $(u, m)$, where $u$ is a morphism of cdga's $A \longrightarrow \Gamma_{n}(B)$ (i.e. $u \in \operatorname{Spec} A(B)$ ), and $m$ is a degree 0 element in $M \otimes_{A} \Gamma_{n}(B)$ (i.e. $m$ is a morphism of complexes 
of $\mathbb{C}$-vector spaces $\left.m: \mathbb{C} \longrightarrow M \otimes_{A} \Gamma_{n}(B)\right)$. This gives a simplicial set $[n] \mapsto \widetilde{M}(B)_{n}$, and therefore defines an object in $D-A f f^{\sim}$

$$
\begin{aligned}
\widetilde{M}: C D G A & \longrightarrow S S e t \\
B & \mapsto \widetilde{M}(B) .
\end{aligned}
$$

Clearly, the projection $(u, m) \mapsto u$ in the notation above induces a morphism $\widetilde{M} \longrightarrow S p e c A$. Finally, this object is endowed in an obvious way with a structure of $\mathcal{O}_{\text {Spec }} A_{\text {-module. }}$

This construction, $M \mapsto \widetilde{M}$ induces a functor

$$
\widetilde{M}: A-\operatorname{Mod} \longrightarrow \mathcal{O}_{\text {Spec } A}-\operatorname{Mod}
$$

from the category of (unbounded) dg-A-modules, to the category of $\mathcal{O}_{\text {Spec } A \text {-modules. This functor }}$ can be derived (by taking first cofibrant replacements of both $A$ and $M$ ) to a functor

$$
\mathbb{R} \widetilde{M}: \operatorname{Ho}(A-M o d) \longrightarrow \operatorname{Ho}\left(\mathcal{O}_{\mathbb{R} S p e c} A-M o d\right) .
$$

Lemma 4.4 The functor $\mathbb{R} \widetilde{M}$ defined above is fully faithful.

Definition 4.5 1. A $\mathcal{O}$-module on a representable D-stack $\mathbb{R} S p e c$ A is called pseudo-quasi-coherent if it is equivalent to some $\mathbb{R} \widetilde{M}$ as above.

2. Let $F$ be a $D$-stack, and $\mathcal{M}$ be a $\mathcal{O}$-module. We say that $\mathcal{M}$ is pseudo-quasi-coherent if for any morphism $u: \mathbb{R} S$ pec $A \longrightarrow F$, the pull-back $u^{*} \mathcal{M}$ is a pseudo-quasi-coherent $\mathcal{O}$-module on $\mathbb{R} S$ pec $A$.

The construction $M \mapsto \widetilde{M}$ described above also has a dual version, denoted by $M \mapsto S p e l(M)$ and defined in a similar way.

Let $A$ be a cdga and $M$ be an (unbounded) dg- $A$-module. For a cdga $B$ and an integer $n$, we define $\operatorname{Spel}(M)(B)_{n}$ to be the set of pairs $(u, \alpha)$, where $u: A \longrightarrow \Gamma_{n}(B)$ is a morphism of cdga, and $\alpha: M \longrightarrow \Gamma_{n}(B)$ is a morphism of dg-A-modules. This defines a $D$-stack $B \mapsto \operatorname{Spel}(M)(B)$ which has a natural projection $(u, \alpha) \mapsto u$, to $\operatorname{Spec} A$. Once again, Spel $(M)$ comes equipped with a natural structure of $\mathcal{O}_{\text {Spec }} A_{\text {-module. Also, this } S p e l}$ construction can be derived, to get a functor

$$
\mathbb{R} \text { Spel }: \operatorname{Ho}(A-M o d)^{o p} \longrightarrow \operatorname{Ho}\left(\mathcal{O}_{\mathbb{R} S p e c} A-M o d\right) .
$$

Lemma 4.6 The functor $\mathbb{R}$ Spel defined above is fully faithful.

Definition 4.7 1. A $\mathcal{O}$-module on a representable D-stack $\mathbb{R} S p e c A$ is called representable if it is equivalent to some $\mathbb{R} \operatorname{Spel}(M)$ as above.

2. Let $F$ be a D-stack, and $\mathcal{M}$ be a $\mathcal{O}$-module. We say that $\mathcal{M}$ is representable or is a linear $D$-stack over $F$ if for any morphism $u: \mathbb{R}$ Spec $A \longrightarrow F$, the pull-back $u^{*} \mathcal{M}$ is a representable $\mathcal{O}$-module on $\mathbb{R}$ Spec $A$.

3. A perfect $\mathcal{O}$-module on a $D$-stack $F$ is a $\mathcal{O}_{F}$-module which is both pseudo-quasi-coherent and representable.

One can prove that the homotopy category of perfect $\mathcal{O}$-modules on $\mathbb{R} \operatorname{Spec} A$ is naturally equivalent to the full sub-category of $\operatorname{Ho}(A-M o d)$ consisting of strongly dualizable modules, or equivalently of $\mathrm{dg}$ - $A$-modules which are retracts of finite cell modules (in the sense of [Kr-Ma, §III.1]). In particular, if $A$ is concentrated in degree 0 , then the homotopy category of perfect $\mathcal{O}$-modules on $\mathbb{R} S p e c A$ is naturally equivalent to the derived category of bounded complexes of finitely generated projective 
$A$-modules.

This notion of perfect $\mathcal{O}$-modules can be used in order to define the $K$-theory of $D$-stacks. For any $D$-stack $F$, one can consider the homotopy category of perfect $\mathcal{O}$-modules on $F$, that we denote by $D_{\text {Perf }}(F)$. This is a triangulated category having a natural Waldhausen model $W \operatorname{Perf}(F)$, from which one can define the $K$-theory spectrum on the $D$-stack $F$, as $K(F):=K(W \operatorname{Perf}(F))$. The tensor product of $\mathcal{O}$-modules makes $K(F)$ into an $E_{\infty}$-ring spectrum. Of course, when $X$ is a scheme $K(i X)$ is naturally equivalent to the $K$-theory spectrum of $X$ as defined in [TT].

A related problem is that of defining reasonable Chow groups and Chow rings for strongly geometric $D$-stacks, receiving Chern classes from the $K$-theory defined above. We are not aware of any such constructions nor we have any suggestion on how to approach the question. It seems however that an intersection theory over $D$-stacks would be a very interesting tool, as it might for example give new interpretations (and probably extensions) of the notion of virtual fundamental class defined in [Be-Fa]. For this case, the idea would be that for any strongly geometric $D$-stack $F$, there exists a virtual fundamental class in the Chow group of its truncation $h^{0} F$. The structural sheaf of $F$ should give rise, in the usual way, to a fundamental class in its Chow group, such that integrating against it over the entire $F$ is the same thing as integrating on its truncation $h^{0} F$ against the virtual fundamental class. However, even if there is not yet a theory of Chow groups for $D$-stacks, if one is satisfied with working with $K$-theory instead of Chow groups, the obvious class $1=:\left[\mathcal{O}_{F}\right] \in K_{0}(F)$, will correspond exactly to the class of the expected virtual structure sheaf.

\subsection{Tangent $D$-stacks}

Let $\operatorname{Spec} \mathbb{C}[\epsilon]$ be the spectrum of the dual numbers, and let us consider $i \operatorname{Spec} \mathbb{C}[\epsilon] \in \operatorname{Ho}\left(D-A f f^{\sim}\right)$.

Definition 4.8 The tangent $D$-stack of a $D$-stack $F$ is defined to be

$$
\mathbb{R} T F:=\mathbb{R} \mathcal{H O M}(i \operatorname{Spec} \mathbb{C}[\epsilon], F) \in \operatorname{Ho}\left(D-A f f^{\sim}\right) .
$$

Note that the zero section morphism Spec $\mathbb{C} \longrightarrow \operatorname{Spec} \mathbb{C}[\epsilon]$ and the natural projection $\operatorname{Spec} \mathbb{C}[\epsilon] \longrightarrow$ Spec $\mathbb{C}$ induces natural morphisms

$$
\pi: \mathbb{R} T F \longrightarrow F \quad e: F \longrightarrow \mathbb{R} T F
$$

where $e$ is a section of $\pi$.

An important remark is that for any $D$-stack $F$, the truncation $h^{0} \mathbb{R} T F$ is equivalent to the tangent stack of $h^{0} F$ (in the sense of [La-Mo, $\left.\S 17\right]$ ). In other words, one has

$$
h^{0} \mathbb{R} T F \simeq T\left(h^{0} F\right) .
$$

In particular, the $D$-stacks $\mathbb{R} T F$ and $i T\left(h^{0} F\right)$ have the same classical points. However, it is not true in general that $i T F \simeq \mathbb{R} T(i F)$ for a stack $F$. Even for a scheme $X$, it is not true that $\mathbb{R} T(i X) \simeq i T X$, except when $X$ is smooth.

Definition 4.9 If $x:$ iSpec $\mathbb{C} \longrightarrow F$ is a point of a $D$-stack $F$, then the tangent $D$-stack of $F$ at $x$ is the homotopy fiber of $\pi: \mathbb{R} T F \longrightarrow F$ at the point $x$. It is denoted by

$$
\mathbb{R} T F_{x}:=\mathbb{R} T F \times_{F}^{h} i \operatorname{Spec} \mathbb{C} \in \operatorname{Ho}\left(D-A f f^{\sim}\right) .
$$

Let us now suppose that $F$ is a strongly geometric $D$-stack. One can show that $\mathbb{R} T F$ is also strongly geometric. In particular, for any point $x$ in $F(\mathbb{C})$ the $D$-stack $\mathbb{R} T F_{x}$ is strongly geometric. 
Actually much more is true. For any strongly geometric $D$-stack $F$, and any point $x$ in $F(\mathbb{C})$, the $D$-stack $\mathbb{R} T F_{x}$ is a linear $D$-stack (over $i$ Spec $\mathbb{C}$ ) as defined in 4.7 . Let us recall that this implies the existence of a natural complex $\mathbb{R} \Omega_{F, x}^{1}$ of $\mathbb{C}$-vector spaces (well defined up to a quasi-isomorphism and concentrated in degree $]-\infty, 1]$ ), with the property that, for any cdga $A$, there exists a natural equivalence

$$
\mathbb{R} T F_{x}(A) \simeq \underline{\mathbb{H o m}}_{C(\mathbb{C})}\left(\mathbb{R} \Omega_{F, x}^{1}, A\right),
$$

where $\mathbb{R} \underline{\operatorname{Hom}}_{C(\mathbb{C})}$ denotes the mapping space in the model category of (unbounded) complexes of $\mathbb{C}$-vector spaces. Symbolically, one writes

$$
\mathbb{R} T F_{x}=\left(\mathbb{R} \Omega_{F, x}^{1}\right)^{*},
$$

where $\left(\mathbb{R} \Omega_{F, x}^{1}\right)^{*}$ is the dual complex to $\mathbb{R} \Omega_{F, x}^{1}$. In other words, the tangent $D$-stack of $F$ at $x$ "is" the complex $\left(\mathbb{R} \Omega_{F, x}^{1}\right)^{*}$, which is now concentrated in degree $[-1, \infty[$.

Definition 4.10 If $x:$ SSpec $\mathbb{C} \longrightarrow F$ is a point of a strongly geometric D-stack, then we say that the dimension of $F$ at $x$ is defined if the complex $\mathbb{R} \Omega_{F, x}^{1}$ has bounded and finite dimensional cohomology. If this is the case, the dimension of $F$ at $x$ is defined by

$$
\mathbb{R} \operatorname{Dim}_{x} F:=\sum_{i}(-1)^{i} H^{i}\left(\mathbb{R} \Omega_{F, x}^{1}\right) .
$$

This linear description of $\mathbb{R} T F_{x}$ has actually a global version. First observe that the $\mathbb{R} S p e l$ construction described in the previous subsection extends, via some gluing process, to a construction $\mathbb{R}$ Spel that can be applied to any pseudo-quasi-coherent module over a geometric D-stack. Then, one can define a cotangent complex $\mathbb{R} \Omega_{F}^{1}$ of a strongly geometric $D$-stack, which is in general an $\mathcal{O}$-module on $F$ in the sense of Definition 4.5, which is most of the times pseudo-quasi-coherent. One then shows that there exists an equivalence of $D$-stacks over $F$

$$
\mathbb{R} T F \simeq \mathbb{R} \underline{\operatorname{Spel}}\left(\mathbb{R} \Omega_{F}^{1}\right),
$$

and in particular that the $D$-stack $\mathbb{R} T F$ is a linear stack over $F$ in the sense of Definition 4.7.

An already interesting application of this description, is to the case $F=i X$, for $X$ a scheme or even an algebraic stack. Indeed, the cotangent complex $\mathbb{R} \Omega_{i X}^{1}$ mentioned above is precisely the cotangent complex $\mathbb{L}_{X}$ of $[\mathrm{La}-\mathrm{Mo}, \S 17]$ and the equivalence

$$
\mathbb{R} T(i X) \simeq \mathbb{R} \underline{\operatorname{Spel}}\left(\mathbb{R} \Omega_{i X}^{1}\right)
$$

gives then a relation between the purely algebraic object $\mathbb{L}_{X}$ and the geometric object $\mathbb{R} T(i X)$. In a sense, the usual geometric intuition about the tangent space is recovered here, at the price of (and thanks to) enlarging the category of objects under study: the cotangent complex of a scheme becomes the derived tangent space of the scheme considered as a $D$-stack. We like to see this as a possible answer to the following remark of A. Grothendieck ([Gr, p. 4]):

[...] Il est très probable que cette théorie pourra s'étendre de façon à donner une correspondance entre complexes de chaines de longeur n, et certaines " $n$-catégories" cofibrées sur $\underline{C}$; et il n'est pas exclus que par cette voie on arrivera également à une "interprétation géométrique" du complexe cotangent relatif de Quillen. 


\subsection{Smoothness}

To finish this part, we investigate various non-equivalent natural notions of smoothness for geometric $D$-stacks.

Strong smoothness. We have already defined the notion of a strongly smooth morphisms of cdga's in $\S 4.1$. We will therefore say that a morphism

$$
F \longrightarrow \mathbb{R} \operatorname{Spec} B
$$

from a geometric $D$-stack $F$ is strongly smooth if there is a strongly smooth atlas $\coprod \mathbb{R} S p e c A_{i} \longrightarrow F$ as in Definition 4.1, such that all the induced morphisms of cdga's $B \longrightarrow A_{i}$ are strongly smooth morphisms of cdga's (see $\S 4.1$ ). More generally, a morphism between strongly geometric $D$-stacks, $F \longrightarrow F^{\prime}$, is called strongly smooth if for any morphism $\mathbb{R} S$ pec $B \longrightarrow F^{\prime}$ the morphism $F \times_{F^{\prime}}^{h}$ $\mathbb{R} S$ pec $B \longrightarrow \mathbb{R} S$ pec $B$ is strongly smooth in the sense above.

Strong smoothness is not very interesting for $D$-stacks, as a strongly geometric $D$-stack $F$ will be strongly smooth if and only if it is of the form $i F^{\prime}$, for $F^{\prime}$ a smooth algebraic stack.

Standard smoothness. A more interesting notion is that of standard smooth morphisms, or simply smooth morphisms. On the level of cdga's they are defined as follows.

A morphism of cdga's $A \longrightarrow B$ is called standard smooth (or simply smooth), if there exists an étale covering $B \longrightarrow B^{\prime}$, and a factorization

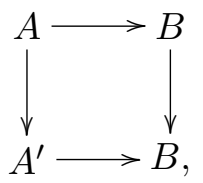

such that the $A$-algebra $A^{\prime}$ is equivalent $A \otimes L(E)$, where $L(E)$ is the free cdga over some bounded complex of finite dimensional $\mathbb{C}$-vector spaces $E$. This notion, defined on cdga's, can be extended (as we did above for strongly smooth morphisms) to morphisms between strongly geometric $D$-stacks.

This notion is more interesting than strong smoothness, as a strongly geometric $D$-stack can be smooth without being an algebraic stack. However, one can check that if $F$ is a smooth strongly geometric $D$-stack in this sense, then $h^{0}(F)$ is also a smooth algebraic stack. In particular, the derived version of the stack of vector bundles on a smooth projective surface, discussed in the Introduction (see also conjecture 5.4), will never be smooth in this sense as its truncation is the stack of vector bundles on the surface which is singular in general).

Nevertheless, smooth morphisms can be used in order to define the following more general notion of geometric $D$-stacks.

Definition 4.11 A D-stack $F$ is 1-geometric if it satisfies the following two conditions

1. The D-stack has a representable diagonal.

2. There exists representable D-stacks $\mathbb{R} S p e c A_{i}$, and a covering

$$
\coprod_{i} \mathbb{R} S \operatorname{Spec} A_{i} \longrightarrow F
$$

such that each of the morphisms $\mathbb{R} S p e c A_{i} \longrightarrow F$ is smooth. Such a family of morphisms will be called $a$ smooth atlas of $F$. 
Essentially all we have said about strongly geometric $D$-stacks is also valid for geometric $D$-stacks in the above sense. The typical example of a geometric $D$-stack which is not strongly geometric is $B G$, where $G$ is a representable group $D$-stack which is not a scheme. For example, one can take $G$ to be of the from $\mathbb{R} \operatorname{Spel}(M)$ for a non-positively graded bounded complex of finite dimensional vector spaces. Then, $G$ is a representable $D$-stack (it is precisely $\mathbb{R} \operatorname{Spec} L(M)$, where $L(M)$ is the free cdga on $M)$, and $B G$ is naturally equivalent to $\mathbb{R} \operatorname{Spel}(M[-1])$. When $M[-1]$ has non-zero $H^{1}$ then $B G$ is not representable anymore but is 1-geometric for the above definition.

More generally, the definition above allows one to consider quotient $D$-stacks $[X / G]$, where $X$ is a representable $D$-stack and $G$ is a smooth representable group $D$-stack acting on $X$.

fp-smoothness. The third notion of smoothness is called fp-smoothness and is the weakest of the three and it seems this is the one which is closer to the smoothness notion referred to in the Derived Deformation Theory program in general (as outlined in [Ko1] or in the introduction to [Ci-Ka1]). It is also well suited in order for the derived stack of vector bundles to be smooth.

Recall that a morphism of cdga's, $A \longrightarrow B$ is finitely presented if it is equivalent to a retract of a finite cell $A$-algebra, or equivalently if the mapping space $\operatorname{Map}_{A / C D G A}(B,-)$ commutes with filtered colimits (this is the same as saying that $\mathbb{R} S p e c A$ commutes with filtered colimits). We will then say that a morphism of geometric $D$-stacks, $F \longrightarrow F^{\prime}$ is locally finitely presented if for any morphism $\mathbb{R}$ Spec $A \longrightarrow F^{\prime}$ there exists a smooth atlas

$$
\coprod \mathbb{R} S \text { Spec } A_{i} \longrightarrow F \times{ }_{F^{\prime}} \mathbb{R} \text { Spec } A
$$

such that all the induced morphisms of cdga's $A \longrightarrow A_{i}$ are finitely presented. Locally finitely presented morphisms will also be called fp-smooth morphisms. The reason for this name is given by the following observation.

Proposition 4.12 Le $F$ be a geometric D-stack which is fp-smooth (i.e. $F \longrightarrow *=i$ Spec $\mathbb{C}$ is fp-smooth). Then the cotangent complex $\mathbb{R} \Omega_{F}^{1}$ is a perfect complex of $\mathcal{O}$-modules on $F$.

In particular, for any point $x \in F(\mathbb{C})$, the dimension of $F$ at $x$ is defined and locally constant for the étale topology.

Of course, one has strongly smooth $\Rightarrow$ smooth $\Rightarrow f p$-smooth, but each of these implications is strict. For example, a smooth scheme is strongly smooth. Let $E$ be a complex in non-positive degrees which is cohomologically bounded and of finite dimension. Then $\mathbb{R} \operatorname{Spel}(E)$ is smooth but not strongly smooth as it is not a scheme in general. Finally, any scheme which is a local complete intersection is fp-smooth, but not smooth in general.

\section{$5 \quad$ Further examples}

In this Section we present three examples of geometric $D$-stacks: the derived stack of local systems on a space, the derived stack of vector bundles and the derived stack of associative algebra and $A_{\infty}$-categorical structures. The derived moduli space of local systems on a space has already been introduced and defined in [Ka2] as a dg-scheme. In the same way, the derived moduli space of (commutative) algebra structures has been constructed in [Ci-Ka2] also as a dg-scheme. Finally, the formal derived moduli spaces of local systems on a space and of $A_{\infty}$-categorical structures have been considered in [Hin2, Ko2, Ko-So].

The new mathematical content of this part is the following. First of all we give a construction of the derived moduli stack of vector bundles, that seems to be new, and we also define global versions of the formal moduli spaces of $A_{\infty}$-categorical structures that were apparently not known. We also provide explicit modular descriptions, by defining various derived moduli functors, which were not known (and probably not easily available), for the constructions of [Ka2, Ci-Ka1, Ci-Ka2]. 


\subsection{Local systems on a topological space}

Throughout this subsection, $X$ will be a CW-complex. For any cdga $A$, we denote by $A-\operatorname{Mod}_{X}$ the category of presheaves of $\operatorname{dg}-A$-modules over $X$. We say that a map $\mathcal{M} \rightarrow \mathcal{N}$ in $A-M o d_{X}$ is a quasi-isomorphism if it induces a quasi-isomorphism of $\mathrm{dg}$ - $A$-modules on each stalk. This gives a notion of equivalences in the category $A-\operatorname{Mod}_{X}$, and of equivalent objects (i.e. objects which are isomorphic in the localization of the category with respect to equivalences).

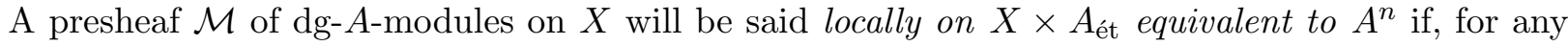
$x \in X$, there exists an open neighborhood $U$ of $x$ in $X$ and an étale cover $A \rightarrow B$, such that the presheaves of dg-B-modules $\mathcal{M}_{\mid U} \otimes_{A} B$ and $B^{n}$ are equivalent. We will also say that a presheaf $\mathcal{M}$ of $\mathrm{dg}$ - $A$-modules is flat, if for any open $U$ of $X$, the $\mathrm{dg}-A$-module $\mathcal{M}(U)$ is cofibrant (for example in the model structure on the category of $d g$ - $A$-modules described in [Hin1]). By composing with a cofibrant replacement functor in $A-M o d$, one can associate to any $\operatorname{dg}-A$-module an equivalent flat $\operatorname{dg}-A$-module (since equivalences are stable by filtered colimits). The category $w \operatorname{Loc}_{n}(X ; A)$ of rank $n$ local systems of $d g$-A-modules has objects those presheaves of flat $\mathrm{dg}$ - $A$-modules on $X$ which are locally on $X \times A_{\text {ét }}$ equivalent to $A^{n}$, and morphisms quasi-isomorphisms between them. For morphisms of cdga's $A \longrightarrow B$ we obtain pull-back functors

$$
\begin{array}{cl}
w \operatorname{Loc}_{n}(X ; A) & \longrightarrow w \operatorname{Loc}_{n}(X ; B) \\
\mathcal{M} & \mapsto \mathcal{M} \otimes_{A} B .
\end{array}
$$

This makes $w \operatorname{Loc}_{n}(X ; A)$ into a lax functor from $C D G A$ to categories, that we turn into a strict functor by applying the standard strictification procedure.

We denote by $\mathbb{R} \underline{L o c} n(X)$ the simplicial presheaf on $D-A f f$ sending a cdga $A$ to $\left|w \operatorname{Loc}_{n}(X)\right|$ (the nerve of $\left.w \operatorname{Loc}_{n}(X ; A)\right)$. We call it the $D$-pre-stack of rank $n$ derived local systems on $X$.

Obviously, the objects in $w \operatorname{Loc}_{n}(X ; A)$ are a derived version of the usual local systems of $R$-modules on $X$, where $R$ is a commutative ring. More precisely, if we consider such an $R$ as a cdga concentrated in degree zero, then $R \operatorname{Loc}_{n}(X ; R)$ is the closure under quasi-isomorphisms of the groupoid of rank $n$ local systems of $R$-modules on $X$; in other words, if we invert quasi-isomorphisms in the category $w \operatorname{Loc}_{n}(X ; R)$ then we obtain a category which is equivalent to the groupoid of rank $n$ local systems of $R$-modules on $X$.

Theorem 5.1 1. The D-pre-stack $\mathbb{R} \underline{\operatorname{Loc}}_{n}(X)$ is a D-stack. Furthermore, one has $\mathbb{R} \underline{L} \underline{L c}_{n}(p t) \simeq$ $i B G l_{n}$.

2. One has an equivalence

$$
h^{0} \mathbb{R} \underline{L o c}_{n}(X) \simeq\left[\operatorname{Hom}\left(\pi_{1}(X), G l_{n}\right) / G l_{n}\right],
$$

between the truncation of $\mathbb{R} \underline{\operatorname{Loc}} n(X)$ and the (Artin) stack of local systems on $X$.

3. If $S(X)$ denotes the singular complex of $X$, we have the following isomorphisms in $\operatorname{Ho}(D-$ Aff $\sim$,

$$
\mathbb{R} \underline{\operatorname{Loc}}{ }_{n}(X) \simeq \mathbb{R} \mathcal{H O M}\left(\underline{S(X)}, i B G l_{n}\right) \simeq \mathbb{R} \mathcal{H O} \mathcal{M}\left(\underline{S(X)}, \operatorname{Loc}_{n}(\mathrm{pt})\right),
$$

where $\mathbb{R} \mathcal{H O M}$ denotes the Hom-stack (internal Hom in $\mathrm{Ho}\left(D-A f f^{\sim}\right)$ ) and $S(X)$ denotes the simplicial constant presheaf with value $S(X)$.

4. For any rank $n$ local system $L$ on $X$, the tangent $D$-stack of $\mathbb{R} L o c_{n}(X)$ at $L$ is the complex $C^{*}(X, \underline{E n d}(L))[1]$, of cohomology of $X$ with coefficients in $\underline{\operatorname{End}}(L)$.

5. If $X$ is a finite $C W$-complex, then the stack $\mathbb{R} \underline{\underline{L}{ }_{n}}(X)$ is strongly geometric, fp-smooth of (the expected) dimension $-n^{2} \chi(X), \chi(X)$ being the Euler characteristic of $X$. 
Note that the classical points of $\mathbb{R} \underline{L o c}_{n}(X)$ (i.e. morphisms from $i$ Speck, for some commutative ring $k$ ) coincide with the classical points of its truncation $h^{0} \mathbb{R} \underline{L o c} n(X)$ which coincides with the usual (i.e. not derived) stack of rank $n$ local systems on $X$. So we have no new classical points, as desired.

Let us give only some remarks to show what the proof of Theorem 5.1 really boils down to. First of all notice that the first assertion is a consequence of the second one, once one knows that $\mathbb{R} \underline{L o c_{n}}(\mathrm{pt}) \simeq i B G L_{n}$ and is a stack; so we are reduced to prove the absolute case $(X=\mathrm{pt})$ of 1 . and 2. The first two properties in 3. follows from 2., the finiteness of $X$ and the analogous properties of $B G l_{n}$. Finally the dimension count in 3 . is made by an explicit computation of the tangent $D$-stack at some local system $E$. Explicitly, one finds that (in the notations of $\S 4.3)\left(\mathbb{R} \Omega_{\mathbb{R} L o c_{n}(X), E}^{1}\right)^{*}$ is the complex $C^{*}(X, \underline{\operatorname{End}}(E))[1]$, which is a complex of $\mathbb{C}$-vector spaces concentrated in degrees $[-1, \infty[$ whose Euler characteristic is exactly $-n^{2} \chi(X)$.

Remark 5.2 The example of local systems is one of those cases where there is a canonical way to derive the usual moduli stack (see the discussion in Section 6). In fact, in this case we have $\mathcal{H O M}\left(S(X), \operatorname{Loc}_{\mathrm{n}}(\mathrm{pt})\right) \simeq \operatorname{Loc}_{n}(X)$, for any CW-complex $X$, where $\mathcal{H O} \mathcal{M}$ denotes the (underived) Hom-stack between (underived) stacks; therefore the natural thing to do is to first view the usual absolute stack $\operatorname{Loc}_{n}(p t)$ as a derived stack via the inclusion $i$ and then derive the Hom-stack from $S(X)$ to $i \operatorname{Loc}_{n}$. This automatically gives an extension of $\operatorname{Loc}_{n}(X)$ i.e. a canonical derivation of it.

It is important to notice that the $D$-stack $\mathbb{R} \underline{\operatorname{Loc}} n(X)$ might be non-trivial even if $X$ is simply connected. Indeed, the tangent at the unit local system is always the complex $C^{*}(X, \mathbb{C})[1]$. This shows that $\mathbb{R} \underline{L} \underline{L c}_{n}(X)$ contains interesting information concerning the higher homotopy type of $X$. As noticed in the Introduction of [K-P-S], this is one of the reasons why the $D$-stack $\mathbb{R} \underline{\operatorname{Loc}}{ }_{n}(X)$ might be an interesting object in order to develop a version of non-abelian Hodge theory. We will therefore ask the same question as in [K-P-S].

Question 5.3 Let $X$ be a smooth projective complex variety and $X^{\text {top }}$ its underlying topological space. Can one extend the non-abelian Hodge structure defined on the moduli space of local systems in [S2], to some kind of Hodge structure on the whole $\mathbb{R} \underline{L o c}_{n}(X)$ ?

This question is of course somewhat imprecise, and it is not clear that the object $\mathbb{R} \underline{L o c} n(X)$ itself could really support an interesting Hodge structure. However, we understand the previous question in a much broader sense, as for example it includes the question of defining derived versions of the moduli spaces of flat and Higgs bundles, and to study their relations from a non-abelian Hodge theoretic point of view, as done for example in [S2].

\subsection{Vector bundles on a projective variety}

We now turn to the example of the derived stack of vector bundles, which is very close to the previous one. Let $X$ be a fixed smooth projective variety.

If $A$ is a cdga, we consider the space $X$ (with the Zariski topology) together with its presheaf of cdga $\mathcal{O}_{X} \otimes A$. It makes sense to consider also presheaves of $\operatorname{dg}-\mathcal{O}_{X} \otimes A$-modules on $X$ and morphisms between them. We define a notion of equivalences between such presheaves, by saying the $f: \mathcal{M} \longrightarrow \mathcal{N}$ is an equivalence if it induces a quasi-isomorphism at each stalks. Using this notion of equivalences we can talk about equivalent $\operatorname{dg}-\mathcal{O}_{X} \otimes A$-modules (i.e. objects which become isomorphic in the localization of the category with respect to quasi-isomorphisms).

We say that a presheaf of $\operatorname{dg}-\mathcal{O}_{X} \otimes A$-module $\mathcal{M}$ on $X$ is a vector bundle of rank $n$, if locally on $X_{z a r} \times A_{\text {ét }}$ it is equivalent to $\left(\mathcal{O}_{X} \otimes A\right)^{n}$ (see the previous Subsection for details on this definition). We consider the category $w V e c t_{n}(X, A)$, of $\operatorname{dg}-\mathcal{O}_{X} \otimes A$-modules which are vector bundles of rank $n$ 
and flat (i.e. for each open $U$ in $X$, the $\mathcal{O}_{X}(U) \otimes A$-module $\mathcal{M}(U)$ is cofibrant), and equivalences between them. By the standard strictification procedure we obtain a presheaf of categories

$$
\begin{array}{ccc}
C D G A & \longrightarrow & C a t \\
A & \mapsto & w V e c t_{n}(X, A) \\
(A \rightarrow B) & \mapsto & \left(\mathcal{M} \mapsto \mathcal{M} \otimes_{A} B\right) .
\end{array}
$$

We then deduce a simplicial presheaf by applying the nerve construction

$$
\begin{array}{rlc}
\mathbb{R} \underline{\operatorname{Vect}}_{n}(X): \quad C D G A & \longrightarrow & \operatorname{SSet} \\
A & \mapsto & \mid \text { wVect }_{n}(X, A) \mid .
\end{array}
$$

This gives an object $\mathbb{R} \underline{V e c t}_{n}(X) \in D-A f f^{\sim}$ that we call the derived moduli stack of rank $n$ vector bundles on $X$.

We state the following result as a conjecture, as we have not checked all details. However, we are very optimistic about it, as we think that a proof will probably consist of reinterpreting the constructions of [Ci-Ka1] in our context.

Conjecture 5.4 1. The D-pre-stack $\mathbb{R} \underline{V e c t}_{n}(X)$ is a strongly geometric, fp-smooth D-stack.

2. There exists a natural isomorphism in $\operatorname{Ho}\left(D-A f f^{\sim}\right)$

$$
\mathbb{R} \underline{\operatorname{Vect}}_{n}(X) \simeq \mathbb{R} \mathcal{H O} \mathcal{M}\left(i X, i B G l_{n}\right) .
$$

3. One has an equivalence

$$
h^{0} \mathbb{R} \underline{\operatorname{Vect}}_{n}(X) \simeq \underline{\operatorname{Vect}}_{n}(X)
$$

between the truncation of the D-stack $h^{0} \mathbb{R} \underline{V e c t}_{n}(X)$ and the (Artin) stack of rank $n$ vector bundles on $X$.

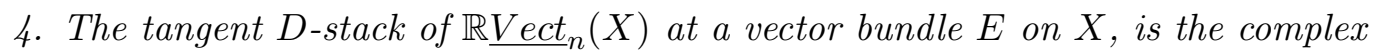

$$
C^{*}\left(X_{Z a r}, \underline{E n d}(E)\right)[1] .
$$

The same remark as in the case of the derived stack of local systems holds. Indeed, the usual Artin stack of vector bundles on $X$ is given by $\mathbb{R} \mathcal{H O M}\left(X, B G l_{n}\right)$, and our $D$-stack of vector bundles on $X$ is $\mathbb{R} \mathcal{H O} \mathcal{M}\left(i X, i B G l_{n}\right)$.

\subsection{Algebras and $A_{\infty}$-categorical structures}

In this last Subsection we present the derived moduli stack of associative algebra structures and $A_{\infty^{-}}$ categorical structures. These are global versions of the formal moduli spaces studied in [Ko2, Ko-So].

Associative algebra structures. We are going to construct a $D$-stack $\mathbb{R} A s s$, classifying associative $d g$-algebra structures.

Let $A$ be any cdga, and let us consider the category of (unbounded) associative differential graded $A$-algebras $A-A s s$ (i.e. $A-A s s$ is the category of monoids in the symmetric monoidal category $A-M o d$, of (unbounded) dg- $A$-modules) $)^{5}$. This category is a model category for which the weak equivalences are the quasi-isomorphisms and fibrations are epimorphisms. We restrict ourselves to

\footnotetext{
${ }^{5}$ By definition our associative $A$-dga's are then all central over $A$ since they are commutative monoids in $A-M o d$.
} 
the category of cofibrant objects $A-A s s^{c}$, and consider the sub-category $w A-A s s^{c}$ consisting of equivalences only. If $A \longrightarrow A^{\prime}$ is any morphism of cdga's, then we have pull-back functors

$$
w A-A s s^{c} \stackrel{-\otimes_{A} A^{\prime}}{\longrightarrow} w A^{\prime}-A s s^{c} .
$$

This defines a (lax) functor on the category of cdga's that we immediately strictify by the standard procedure. We will therefore assume that the above constructions are strictly functorial in $A$. By passing to the corresponding nerves we get a presheaf of simplicial sets

$$
\begin{aligned}
& \mathbb{R} A s s: C D G A \quad \longrightarrow \quad \text { SSet } \\
& A \quad \mapsto\left|w A-A s s^{c}\right| .
\end{aligned}
$$

This gives a well defined object $\mathbb{R} A s s$ in $D-A f f^{\sim}$.

We define a sub-simplicial presheaf $\mathbb{R} A s s_{n}$ of $\mathbb{R} A s s$, consisting of associative dg- $A$-algebras $B$ for which there exists an étale covering $A \longrightarrow A^{\prime}$ such that the $\operatorname{dg}-A^{\prime}$-module $B \otimes_{A}^{\mathbb{L}} A^{\prime}$ is equivalent to $\left(A^{\prime}\right)^{n}$.

Theorem 5.5 1. The D-pre-stack $\mathbb{R} A s s_{n}$ is a D-stack.

2. The D-stack $\mathbb{R} A s s_{n}$ is strongly geometric. Furthermore, $h^{0} \mathbb{R} A s s_{n}$ is naturally equivalent to the (usual) Artin stack of associative algebra structures on $\mathbb{C}^{n}$.

3. For any global point $V: * \longrightarrow \mathbb{R} A s s_{n}$, corresponding to an associative $\mathbb{C}$-algebra $V$, the tangent $D$-stack of $\mathbb{R} A s s_{n}$ at $V$ is the complex $\mathbb{R} \operatorname{Der}(V, V)[1]$ of (shifted) derived derivations from $V$ to $V$.

¿From (3) we see that the geometric $D$-stack $\mathbb{R} A s s_{n}$ is not fp-smooth. Indeed, Quillen gives in [Q, Ex. 11.8] an example of a point in $\mathbb{R} A s s_{n}$ at which the dimension in the sense of Definition 4.10 is not defined.

The previous theorem can also be extended in the following way. Let $V$ be a fixed cohomologically bounded and finite dimensional complex of $\mathbb{C}$-vector spaces. We define $\mathbb{R} A s s_{V}$ to be the sub-simplicial presheaf of $\mathbb{R} A s s$ consisting of associative dg- $A$-algebras $B$ for which there exists an étale covering $A \longrightarrow A^{\prime}$ such that the $\mathrm{dg}$ - $A^{\prime}$-module $B \otimes \mathbb{L}_{A} A^{\prime}$ is equivalent to $A^{\prime} \otimes V$.

One can show that $\mathbb{R} A s s_{V}$ is again a $D$-stack, but it is not in general strongly geometric in the sense of Definition 4.1 (nor in the sense of Definition 4.11). However, we would like just to mention that $\mathbb{R} A s s_{V}$ is still geometric in some sense when considered as a stack over unbounded cdga's (the reader will find details in the forthcoming paper [HAG-II]). The tangent $D$-stack of $\mathbb{R} A s s_{V}$ at a point is given by the same formula as before.

The construction of $\mathbb{R} A s s_{V}$ can also be extended to classify algebra structures over an operad on the complex $V$. One can check that the $D$-stacks one obtains in this way are again geometric. These are the geometric counterparts of the (discrete) moduli spaces described by C. Rezk in [Re].

$A_{\infty}$-Categorical structures ${ }^{6}$. Let $A$ by any cdga. Recall that a $d g$-A-category $C$ consists of the following data

1. A set of objects $O b(C)$.

2. For each pair of object $(x, y)$ in $O b(C)$, a (unbounded) dg- $A$-module $C_{x, y}$.

\footnotetext{
${ }^{6}$ We are working here with the stronger notion of dg-category (or strict $A_{\infty}$-categories), and of course one could also use $A_{\infty}$-categories instead. However, as the homotopy theories of dg-categories and of $A_{\infty}$-categories are equivalent, the $D$-stacks obtained would be the same.
} 
3. For each triplet of object $(x, y, z)$ in $O b(C)$, a composition morphism $C_{x, y} \otimes_{A} C_{y, z} \longrightarrow C_{x, z}$ which satisfies obvious associativity and unital conditions.

There is an obvious notion of morphism between $\mathrm{dg}$ - $A$-categories. There is also a notion of equivalences of $\mathrm{dg}$ - $A$-categories: they are morphisms $f: C \longrightarrow C^{\prime}$ satisfying the following two conditions

1. For any pair of objects $(x, y)$ of $C$, the induced morphism $f_{x, y}: C_{x, y} \longrightarrow C_{x, y}^{\prime}$ is a quasiisomorphism of $\mathrm{dg}$ - $A$-modules.

2. Let $H^{0}(C)$ (resp. $H^{0}\left(C^{\prime}\right)$ ) be the categories having respectively the same set of objects as $C$ (resp. as $C^{\prime}$ ), and $H^{0}\left(C_{x, y}\right)$ (resp. $H^{0}\left(C_{x, y}^{\prime}\right)$ ) as set of morphisms from $x$ to $y$. Then, the induced morphism

$$
H^{0}(f): H^{0}(C) \longrightarrow H^{0}\left(C^{\prime}\right)
$$

is an equivalence of categories (in the usual sense).

Using these definitions, one has for any cdga $A$, a category $A-C a t$ of $\operatorname{dg}-A$-categories, with a sub-category of equivalences $w A-C a t$. Furthermore, if $A \longrightarrow A^{\prime}$ is a morphism of cdga, one has a pull-back functor $A-C a t \longrightarrow A^{\prime}-C a t$, obtained by tensoring the $\operatorname{dg}-A$-modules $C_{x, y}$ with $A^{\prime}$. With a bit of care (e.g. by restricting to cofibrant $\operatorname{dg}$ - $A$-categories), one gets a simplicial presheaf

$$
\begin{array}{ccc}
\mathbb{R} C a t: C D G A & \longrightarrow & \text { SSet } \\
A & \mapsto|w A-C a t|,
\end{array}
$$

that is an object in $D-A f f^{\sim}$.

We now fix a graph $\mathcal{O}$ of non-positively graded complexes of $\mathbb{C}$-vector spaces. This means that $\mathcal{O}$ is the datum of a set $\mathcal{O}_{0}$, and for any $(x, y) \in \mathcal{O}$, of a complex $\mathcal{O}_{x, y}$. We will assume that all the complexes $\mathcal{O}_{x, y}$ are bounded with finite dimensional cohomology. We consider the sub-simplicial presheaf $\mathbb{R} C a t_{\mathcal{O}}$ of $\mathbb{R} C a t$, consisting of all those $\operatorname{dg}$ - $A$-categories $C$ such that locally on $A_{\text {ét }}$ the underlying graph of $C$ is equivalent to $\mathcal{O} \otimes A$; the underlying graph of $C$ is defined to be the graph $G(C)$ whose set of objects is a set of representatives of isomorphism classes of objects in $H^{0}(C)$, and whose complexes of morphisms are the ones of $C$. The simplicial presheaf $\mathbb{R} C a t_{\mathcal{O}}$ classifies dg-categorical structures on the graph $\mathcal{O}$.

The following theorem identifies the tangent of $\mathbb{R} C a t_{\mathcal{O}}$.

Theorem 5.6 Let $\widetilde{\mathbb{R C a t}}$ 作 be the associated D-stack to the D-pre-stack $\mathbb{R} C a t_{\mathcal{O}}$. For any global point $C: * \longrightarrow \mathbb{R} C a t_{\mathcal{O}}$, corresponding to a dg-category $C$, the tangent D-stack of $\widetilde{\mathbb{R} C a t_{\mathcal{O}}}$ at $C$ is the whole (shifted) Hochschild cohomology complex $C^{*}(C, C)[2]$ (see e.g. [Ko-So, 2.1] or [So, 2]).

Remark 5.7 For a cdga $A$, points in $\widetilde{\mathbb{R C a t}}(A)$ can be described as certain twisted forms of dg- $A$ categories on the étale site of $A$.

Let us suppose that $\mathcal{O}$ is now a graph of finite dimensional vector spaces (i.e. the complexes $\mathcal{O}_{x, y}$ are concentrated in degree 0 for any $x, y)$. Then one can show that the $D$-stack $\widetilde{\mathbb{R} C a t_{\mathcal{O}}}$ is strongly 2 -geometric. Here we use a notion of strongly $n$-geometric $D$-stacks obtained by iterating Definition 4.1. The reader will find details about higher geometric stacks in [HAG-II] and might also wish to consult $[\mathrm{S} 1]$. Note that the $D$-stack $\widetilde{\mathbb{R} C a t_{\mathcal{O}}}$ cannot be 1 -geometric, as its truncation $h^{0} \widetilde{\mathbb{R} C a t_{\mathcal{O}}}$ is the (2-)stack of linear categories. As a 1-geometric (not derived) stack is always 1-truncated (as opposite to the derived case), this shows that $\widetilde{\mathbb{R C a t}} t_{\mathcal{O}}$ must be at least 2 -geometric.

As in the case of $\mathbb{R} A s s$, if the graph $\mathcal{O}$ is not a graph of vector spaces, then the $D$-stack $\widetilde{\mathbb{R} C a t_{\mathcal{O}}}$ is not strongly 2-geometric anymore, but is still geometric in some sense, when considered as a stack 
over unbounded cdga's.

Let $V$ be a bounded complex with finite dimensional cohomology, also considered as a graph of complexes with a unique object. Then, there exists a natural morphism

$$
\mathbb{R} A s s_{V} \longrightarrow \widetilde{\mathbb{R C a t}} t_{V}
$$

that sends an associative dga to the dg-category, with one object, it defines. This morphism is actually a gerbe in the following sense. If $B: \mathbb{R} S p e c A \longrightarrow \mathbb{R} A s s_{V}$ corresponds to an associative $A$-dga $B$, then the homotopy fiber $F$ of the previous morphism is locally equivalent to the $D$-stack over $\mathbb{R} S p e c A$ sending a cdga $A \rightarrow A^{\prime}$ to the simplicial set $K\left(\left(B \otimes_{A} A^{\prime}\right)^{*}, 1\right)$, where $\left(B \otimes_{A} A^{\prime}\right)^{*}$ is the loop space of invertible elements in $B \otimes_{A} A^{\prime}$ (i.e. the mapping space $\left.\operatorname{Map}_{A^{\prime}-a l g}\left(A^{\prime}\left[T, T^{-1}\right], B \otimes_{A} A^{\prime}\right)\right)$. In particular, one deduces that the morphism $\mathbb{R} A s s_{V} \longrightarrow \widetilde{\mathbb{R C a t}}$ is a smooth fibration of $D$-stacks. This smooth morphism induces in particular an exact triangle between the tangent $D$-stacks

$$
\mathbb{R} T F_{B} \longrightarrow \mathbb{R} T\left(\mathbb{R} A s s_{V}\right)_{B} \longrightarrow \mathbb{R} T\left(\widetilde{\mathbb{R} C a t_{V}}\right)_{B} \stackrel{+1}{\longrightarrow}
$$

which can also be written

$$
B[1] \longrightarrow \mathbb{R} \operatorname{Der}_{A}(B, B)[1] \longrightarrow C_{A}^{+}(B, B)[2] \stackrel{+1}{\longrightarrow}
$$

which is our way of understanding the triangle appearing in [Ko2, p. 59] (at least for $d=1$ ).

The fact that the tangent $D$-stack of $\widetilde{R C a t} V$ at a dg-category with only one object is the whole (shifted) Hochschild complex $C^{*}(A, A)[2]$, where $A$ is the dg-algebra of endomorphisms of the unique object, is also our way to understand the following sentence from [Ko-So, p. 266].

In some sense, the full Hochschild complex controls deformations of the $A_{\infty}$-category with one object, such that its endomorphism space is equal to $A$.

We see that the previous results and descriptions produce global versions of the formal moduli spaces of $A_{\infty}$-categories studied for example in [Ko-So, So]. This also shows that there are interesting higher geometric stacks, and probably even more interesting examples will be given by the $D$-stack of $(n-1)$-dg-categories (whatever these are) as suggested by a higher analog of the exact triangle above (see [Ko2, 2.7 Claim 2]).

\section{References}

[BeI] K. Behrend, Differential Graded Schemes I: Perfect Resolving Algebras , Preprint math.AG/0212225.

[BeII] K. Behrend, Differential Graded Schemes II: The 2-category of Differential Graded Schemes, Preprint math.AG/0212226.

[Be-Fa] K. Behrend, B. Fantechi, The intrinsic normal cone, Invent. Math. 128 (1997), No. 1, 45-88.

[Bl] B. Blander, Local projective model structure on simplicial presheaves, K-theory 24 (2001) No. 3, 283-301.

[Ci-Ka1] I. Ciocan-Fontanine, M. Kapranov, Derived Quot schemes, Ann. Sci. Ecole Norm. Sup. (4) 34 (2001), 403-440. 
[Ci-Ka2] I. Ciocan-Fontanine, M. Kapranov, Derived Hilbert Schemes, preprint math.AG/0005155.

[De] P. Deligne, Catégories Tannakiennes, in Grothendieck Festschrift Vol. II, Progress in Math. 87, Birkhauser, Boston 1990.

[DHI] D. Dugger, S. Hollander, D. Isaksen, Hypercovers and simplicial presheaves, preprint math.AT/0205027.

[EKMM] A.D. Elmendorf, I. Kriz, M.A. Mandell, J.P. May, Rings, modules, and algebras in stable homotopy theory, Mathematical Surveys and Monographs, vol. 47, American Mathematical Society, Providence, $R I, 1997$.

[Gr] A.Grothendieck, Catégories cofibrées additives et complexe cotangent relatif, Lecture Note in Mathematics 79, Springer-Verlag, Berlin, 1968.

[Ha] M. Hakim, Topos annelés et schémas relatifs, Ergebnisse der Mathematik und ihrer Grenzgebiete, Band 64. Springer-Verlag Berlin-New York, 1972.

[Hin1] V. Hinich, Homological algebra of homotopical algebras, Comm. in Algebra 25 (1997), 32913323 .

[Hin2] V. Hinich, Formal stacks as dg-coalgebras, J. Pure Appl. Algebra 162 (2001), No. 2-3, 209-250.

[Hi] P. S. Hirschhorn, Model Categories and Their Localizations, Mathematical Surveys and Monographs 99, AMS, 2003.

[H-S] A. Hirschowitz, C. Simpson, Descente pour les n-champs, preprint math.AG/9807049.

[Hol] S. Hollander, A homotopy theory for stacks, preprint math.AT/0110247.

[Ho] M. Hovey, Model categories, Mathematical surveys and monographs, Vol. 63, Amer. Math. Soc. Providence 1998.

[Ho-Sh-Sm] M. Hovey, B.E. Shipley, J. Smith, Symmetric spectra, J. Amer. Math. Soc. 13 (2000), no. 1, 149-208.

[Ja1] J. F. Jardine, Simplicial presheaves, J. Pure and Appl. Algebra 47 (1987), 35-87.

[Jo-Ti] A. Joyal, M. Tierney, Strong stacks and classifying spaces, in Category theory (Como, 1990), Lecture Notes in Mathematics 1488, Springer-Verlag New York, 1991, 213-236.

[Ka1] M. Kapranov, dg-Schemes in algebraic geometry, talk at MSRI, March 2002, notes available at http://www.msri.org/publications/In/msri/2002/intersect/kapranov/1/index.html.

[Ka2] M. Kapranov, Injective resolutions of BG and derived moduli spaces of local systems, J. Pure Appl. Algebra 155 (2001), No. 2-3, 167-179.

[Ka-Vo] M. Kapranov, V. Voevodsky, 2-Categories and Zamolodchikov tetrahedra equations, in Algebraic groups and their generalizations: Quantum and infinte dimensional methods, University Park PA, 1991, 177-269, Proc. Sympos. Pure Math. 56 part 2, AMS, Providence, RI, 1994.

[K-P-S] L. Katzarkov, T. Pantev, C. Simspon, Non-abelian mixed Hodge structures, preprint math.AG/0006213.

[Ko1] M. Kontsevich, Enumeration of rational curves via torus actions, The moduli space of curves (Texel Island, 1994), 335-368, Progr. Math. 129, Birkhauser Boston, MA, 1995. 
[Ko2] M. Kontsevich, Operads and motives in deformation quantization, Moshé Flato (1937-1998), Lett. Math. Phys. 48, (1999), No. 1, 35-72.

[Ko-So] M. Kontsevich, Y. Soibelman, Deformations of algebras over operads and the Deligne conjecture, Conférence Moshé Flato 1999, Vol. 1 (Dijon), 255-307, Math. Phys. Stud. 21, Kluwer Acad. Publ, Dordrecht, 2000.

[Kr-Ma] I. Kriz, J. P. May, Operads, algebras, modules and motives, Astérisque 233, 1995.

[La-Mo] G. Laumon and L. Moret-Bailly, Champs algébriques, A series of Modern Surveys in Mathematics vol. 39, Springer-Verlag 2000.

[Man] M. Manetti, Extended deformation functors, Int. Math. Res. Not. 14 (2002), 719-756.

[MCM] R. Mc Carthy, V. Minasian, Smooth S-algebras, preprint 2002.

[Mil] J. S. Milne, Étale cohomology, Princeton University Press, 1980.

[Q] D. Quillen, On the (co-)homology of commutative rings, Applications of Categorical Algebra (Proc. Sympos. Pure Math., Vol XVII, New York, 1964), 65-87. Amer. Math. Soc., Providence, P.I.

[Re] C. Rezk, Spaces of algebra structures and cohomology of operads, Thesis 1996, available at http://www.math.uiuc.edu/ rezk.

[S1] C. Simpson, Algebraic n-stacks, preprint math.AG/9609014.

[S2] C. Simpson, The Hodge filtration on non-abelian cohomology, preprint math.AG/9604005.

[So] Y. Soibelman, Mirror symmetric and deformation quantization, preprint hep-th/0202128.

[TT] R. W. Thomason, T. Trobaugh, Higher algebraic K-theory of schemes and of derived categories, pp. 247-435 in The Grothendieck Festschrift, Vol. III, Birkhäuser, 1990.

[HAG-I] B. Toën, G. Vezzosi, Homotopy Algebraic Geometry I: Topos theory, preprint math.AG/0207028, submitted.

[HAG-II] B. Toën, G. Vezzosi, Homotopy Algebraic Geometry II: Geometric stacks, in preparation.

[Vo] R. M. Vogt, Introduction to algebra over "brave new rings", The 18th winter school "Geometry and physics" (Srni, 1998), Rend. Circ. Mat. Palermo (2) Suppl. 59 (1999), 49-82. 